\title{
On the Analytic Invariants and Semiroots of Plane Branches
}

\author{
Marcelo Osnar Rodrigues de Abreu \\ and \\ Marcelo Escudeiro Hernandes* ${ }^{* \dagger}$
}

April 26, 2021

2010 Mathematics Subject Classification: 14H20 (primary), 32S10 (secondary)

keywords: Plane curves, Semiroots, Analytic Invariants.

\begin{abstract}
The value semigroup of a $k$-semiroot $C_{k}$ of a plane branch $C$ allows us to recover part of the value semigroup $\Gamma=\left\langle v_{0}, \ldots, v_{g}\right\rangle$ of $C$, that is, it is related to topological invariants of $C$. In this paper we consider the set of values of differentials $\Lambda_{k}$ of $C_{k}$, that is an analytical invariant, and we show how it determine part of the set of values of differentials $\Lambda$ of $C$. As a consequence, in a fixed topological class, we relate the Tjurina number $\tau$ of $C$ with the Tjurina number of $C_{k}$. In particular, we show that $\tau \leq \mu-\frac{3 n_{g}-2}{4} \mu_{g-1}$ where $n_{g}=\operatorname{gcd}\left(v_{0}, \ldots, v_{g-1}\right)$, $\mu$ and $\mu_{g-1}$ denote the Milnor number of $C$ and $C_{g-1}$ respectively. If $n_{g}=2$, we have that $\tau=\mu-\mu_{g-1}$ for any curve in the topological class determined by $\Gamma$ that is a generalization of a result obtained by Luengo and Pfister.
\end{abstract}

\section{Introduction}

Given $f \in \mathbb{C}\{x, y\}$ irreducible the equation $f=0$ define a germ of irreducible plane curve, or a branch, $C_{f}$.

The topological class of $C_{f}$ can be totaly characterized by numerical data: by the characteristic exponents of a Puiseux parametrization, the value semigroup $\Gamma_{f}$, the multiplicity sequence in the canonical resolution, for instance. All of these numerical invariants determine each other and can be easily computed.

The value semigroup $\Gamma_{f} \subseteq \mathbb{N}$ admits a conductor $\mu_{f}$ that is, $\mu_{f}-1 \notin \Gamma_{f}$ and $\mu_{f}+\mathbb{N} \subseteq \Gamma_{f}$. So, $\Gamma_{f}$ is determined by the finite set $\mathbb{N} \backslash \Gamma_{f}$ and $\mu_{f}$ is a topological invariant that coincide with the Milnor number of $f$, i.e., $\mu_{f}=\operatorname{dim}_{\mathbb{C}} \frac{\mathbb{C}\{x, y\}}{\left\langle f_{x}, f_{y}\right\rangle}$.

${ }^{*}$ The first author was partially supported by CAPES and the second one by CNPq-Brazil.

${ }^{\dagger}$ Corresponding author: Hernandes, M. E.; email: mehernandes@uem.br 
On the other hand, it is not so easy to compute analytical invariants and to describe relation among them. In [HH3], the set $\Lambda_{f}$ of values of differentials was the principal analytical invariant and ingredient considered to obtain normal forms with respect to the analytical equivalence of plane branches. The set $\Lambda_{f}$ is related with the Tjurina number $\tau_{f}=\operatorname{dim}_{\mathbb{C}} \frac{\mathbb{C}\{x, y\}}{\left\langle f, f_{x}, f_{y}\right\rangle}$, another analytical invariant of $C_{f}$, by the formula $\tau_{f}=\mu_{f}-\sharp\left(\Lambda_{f} \backslash \Gamma_{f}\right)$.

Up to analytical equivalence any plane curve can be defined by a Weierstrass polynomial $f \in \mathbb{C}\{x\}[y]$ and many topological aspects of $C_{f}$ can be studied by a set of monic irreducible polynomials $f_{k} \in \mathbb{C}\{x\}[y], 0 \leq k<g$ with $\operatorname{deg}_{y}\left(f_{k}\right)=\frac{v_{0}}{e_{k}}$, called semiroots of $f$, such that $\nu_{f}\left(f_{k}\right):=\operatorname{dim}_{\mathbb{C}} \frac{\mathbb{C}\{x, y\}}{\left\langle f, f_{k}\right\rangle}=v_{k+1}$ where $\Gamma_{f}=\left\langle v_{0}, v_{1}, \ldots, v_{g}\right\rangle, \operatorname{deg}_{y}(f)=v_{0}=\operatorname{dim}_{\mathbb{C}} \frac{\mathbb{C}\{x, y\}}{\langle f, x\rangle}$ and $e_{i}=\operatorname{gcd}\left(v_{0}, \ldots, v_{i}\right)$ (see Section 2).

Several authors have considered semiroots in different contexts. For instance, Zariski in [Z3] considered semiroots given by minimal polynomial of truncation of Puiseux parametrization of $C_{f}$ to compute the minimal set of generators of the semigroup. Abhyankar in $\mathrm{Ab}$ presented an irreducibility criterion for elements in $\mathbb{C}\{x\}[y]$ using approximate roots that are particular cases of semiroots. In [Y], Yavorski gave a method to construct a miniversal family of polynomials in $\mathbb{C}[x, y]$ that define branches with a fixed semigroup considering semiroots. Popescu in Pop presented several local properties of semiroots including a proof of the Embedding Line Theorem as an application.

It is possible to relate the topological data of $C_{f}$ with the corresponding data of the branch $C_{k}$ associated to $f_{k}$. For example, we have

$$
\Gamma_{f}=e_{k} \Gamma_{k}+\left\langle v_{k+1}, \ldots, v_{g}\right\rangle \quad \text { and } \quad \mu_{f}-1=e_{k}\left(\mu_{k}-1\right)+\sum_{j=k+1}^{g}\left(n_{j}-1\right) v_{j},
$$

where $n_{j}=\frac{e_{j-1}}{e_{j}}, \Gamma_{k}$ is the value semigroup and $\mu_{k}$ is the Milnor number of $C_{k}$.

The aim of this work is to study relations among the analytical invariants $\Lambda_{f}, \tau_{f}, \Lambda_{k}$ and $\tau_{k}$ associated to $C_{f}$ and $C_{k}$ respectively. For this purpose, in Section 3 we explore the logarithmic differential forms along a curve and we revisit results of Briançon, Maisonobe and Torrelli (see [BMT]) about some magic matrix associated to a plane curve and its Jacobian ideal.

In the Section 4 we describe a common subset of $\Lambda \backslash \Gamma$ shared by any plane branch $C$ that admits a fixed semiroot $f_{k}$ and consequently we can relate the Tjurina number $\tau$ of $C$ with the Tjurina number $\tau_{k}$ of $C_{k}$. More explicitly, for $0 \leq k<g$ we get $\tau \leq \mu-\mu_{k}-e_{k+1}\left(n_{k+1}-2\right) \tau_{k}$.

Dimca and Greuel in [DG] conjectured that $\frac{3}{4} \mu \leq \tau$ for any plane curve. This question motivated several works, see [AABM], GH], Wan] for a proof in the irreducible case and [Al] for any plane curve. So, for $k=g-1$ we get $\tau \leq \mu-\mu_{g-1}-\left(n_{g}-2\right) \tau_{g-1} \leq \mu-\left(\frac{3 n_{g}-2}{4}\right) \mu_{g-1}$, that is an upper bound for $\tau$ for any plane branch in a fixed topological class.

Considering the topological class given by $\Gamma=\left\langle v_{0}, \ldots, v_{g}\right\rangle$ with $n_{g}=\operatorname{gcd}\left(v_{0}, \ldots, v_{g-1}\right)=2$, in Section 5 we show that $\tau$ is constant and equal to $\mu-\mu_{g-1}$ for any plane branch that admits the semigroup $\Gamma$. This result is trivial if $g=1$ and it was showed by Luengo and Pfister for $g=2$ in $[\mathrm{LP}]$. 


\section{Plane branches and semiroots}

In what follows we recall some classical result about plane curves using the presentation of [BK], [H] and [Z3].

Let $\mathbb{C}\{x, y\}$ be the ring of analytic power series ring in $x$ and $y$ over $\mathbb{C}$ and $\mathcal{M}=\langle x, y\rangle$ its maximal ideal. Let $C_{f} \in\left(\mathbb{C}^{2}, 0\right)$ be a singular irreducible plane curve (a plane branch) defined by $f=0$, where $f \in \mathcal{M}^{n} \backslash \mathcal{M}^{n+1}$ is irreducible and $n>1$ is its multiplicity.

We say that $C_{f}$ and $C_{h}$ are analytically equivalent if there exist neighborhoods $U, V \subseteq\left(\mathbb{C}^{2}, 0\right)$ and an analytic isomorphism $\Psi:\left(\mathbb{C}^{2}, 0\right) \rightarrow\left(\mathbb{C}^{2}, 0\right)$ such that $\Psi\left(U \cap C_{f}\right)=V \cap C_{h}$. If $\Psi$ is just a homeomorphism we say that $C_{f}$ and $C_{h}$ are topologically equivalent.

By the Weierstrass Preparation Theorem and a change of coordinates if it is necessary, any plane branch is analytically equivalent to a branch defined by $f=y^{n}+\sum_{i=1}^{n} c_{i}(x) y^{n-i} \in \mathbb{C}\{x\}[y]$ with $n \nmid \operatorname{ord}_{x}\left(c_{n}(x)\right)=m$ and by the Newton-Puiseux theorem $C_{f}$ admits primitive 1 Puiseux parametrizations $\left(t^{n}, \varphi\left(\zeta^{j} t\right)\right)$ with $1 \leq j \leq n$ where $\varphi(t)=\sum_{i \geq m} a_{i} t^{i} \in \mathbb{C}\{t\}, a_{m} \neq 0$ and $\zeta$ is a primitive $n$-th root of unity, that is, $f(x, y)=\prod_{j=1}^{n}\left(y-\varphi\left(\zeta^{j} x^{\frac{1}{n}}\right)\right)$.

Given a Puiseux parametrization as above we consider the following sequences of positive integers:

$$
\begin{gathered}
n=\beta_{0}=e_{0}, \quad n_{0}=1 \\
\beta_{i}=\min \left\{k ; a_{k} \neq 0 \text { and } k \not \equiv 0 \bmod e_{i-1}\right\}, \quad e_{i}=\operatorname{gcd}\left(e_{i-1}, \beta_{i}\right), \quad n_{i}=\frac{e_{i-1}}{e_{i}} \quad \text { for } i \geq 1 .
\end{gathered}
$$

As the parametrization is primitive there exists an integer $g>0$ such that $e_{g}=1$. The increasing finite sequence $\beta_{0}<\beta_{1}<\ldots<\beta_{g}$ is called the characteristic sequence. According to Zariski (see [Z1]) the topological class of $C_{f}$ is totaly determined by its characteristic sequence.

The topological data can be done by other numerical invariants. For instance, considering the exact sequence

$$
\begin{aligned}
\{0\} \rightarrow\langle f\rangle \rightarrow \mathbb{C}\{x, y\} & \stackrel{\varphi^{*}}{\rightarrow} \mathbb{C}\left\{t^{n}, \varphi(t)\right\} \rightarrow\{0\} \\
h & \mapsto \varphi^{*}(h):=h\left(t^{n}, \varphi(t)\right)
\end{aligned}
$$

we have $\mathcal{O}_{f}:=\frac{\mathbb{C}\{x, y\}}{\langle f\rangle} \approx \mathbb{C}\left\{t^{n}, \varphi(t)\right\} \subseteq \mathbb{C}\{t\} \approx \overline{\mathcal{O}}_{f}$. The isomorphism class of the local ring $\mathcal{O}_{f}$ determine the analytic class of $C_{f}$ and we can recover the topological data by means $\mathcal{O}_{f}$ considering

$$
\Gamma_{f}=\left\{\nu_{f}(h):=\operatorname{ord}_{t}\left(\varphi^{*}(h)\right)=\operatorname{dim}_{\mathbb{C}} \frac{\mathbb{C}\{x, y\}}{\langle f, h\rangle} ; h \in \mathbb{C}\{x, y\} \backslash\langle f\rangle\right\} \subseteq \mathbb{N} .
$$

In fact, $\Gamma_{f}$ is a numerical semigroup that admits the minimal set of generators $\left\{v_{0}, v_{1}, \ldots, v_{g}\right\}$ satisfying

$$
v_{0}=\beta_{0}, \quad v_{i+1}=n_{i} v_{i}+\beta_{i+1}-\beta_{i}=\sum_{j=1}^{i} \frac{e_{j-1}-e_{j}}{e_{i}} \beta_{j}+\beta_{i+1}, \quad 0 \leq i<g .
$$

\footnotetext{
${ }^{1} \mathrm{~A}$ parametrization $\left(t^{n}, \sum_{i} a_{i} t^{i}\right)$ is primitive if and only if $\operatorname{gcd}\left(n, i ; a_{i} \neq 0\right)=1$.
} 
So, $\Gamma_{f}$ determines and it is determined by the characteristic sequence.

The integer $n_{i}$ can be characterized by the property $n_{i}=\min \left\{k>0 ; k v_{i} \in\left\langle v_{0}, \ldots, v_{i-1}\right\rangle\right\}$.

Remark 2.1. Any positive integer $r$ can be uniquely expressed as $r=\sum_{i=0}^{g} s_{i} v_{i}$ with $0 \leq s_{i}<n_{i}$ for $1 \leq i \leq g$ and $s_{0} \in \mathbb{Z}$. Moreover, $r \in \Gamma_{f}$ if and only if, $s_{0} \geq 0$ (see Lemma \%.1, [H]]).

As $\Gamma_{f}$ is a numerical semigroup it admits a conductor $\mu_{f}$ that is, $\mu_{f}-1 \notin \Gamma_{f}$ and $\mu_{f}+\mathbb{N} \subseteq \Gamma_{f}$. In this case, $\mu_{f}$ coincides with the Milnor number of $C_{f}$ and (see [M] and (7.1) in [H] )

$$
\mu_{f}=\operatorname{dim}_{\mathbb{C}} \frac{\mathbb{C}\{x, y\}}{J(f)}=\sum_{i=1}^{g}\left(n_{i}-1\right) v_{i}-v_{0}+1=n_{g} v_{g}-\beta_{g}-v_{0}+1=2 \sharp(\mathbb{N} \backslash \Gamma),
$$

where $J(f)=\left\langle f_{x}, f_{y}\right\rangle \subseteq \mathbb{C}\{x, y\}$.

Concerning the vector space $\frac{\mathbb{C}\{x, y\}}{J(f)}$, Yavorski presents the following theorem:

Theorem 2.2 (Yavorski, $[\mathbf{Y}]$ ). For any set $\mathcal{B}=\left\{h \in \mathbb{C}\{x\}[y] ; \operatorname{deg}_{y}(h)<\operatorname{deg}_{y}(f)\right\}$ satisfying

1. $\nu_{f}(h) \in \Gamma_{f} \backslash\left\{\mu_{f}-1+\gamma ; \gamma \in \Gamma_{f}\right\}$;

2. $\nu_{f}\left(h_{i}\right) \neq \nu_{f}\left(h_{j}\right)$ for every $h_{i}, h_{j} \in \mathcal{B}$,

we have a $\mathbb{C}$-basis $\overline{\mathcal{B}}=\left\{\bar{h} \in \frac{\mathbb{C}\{x, y\}}{J(f)} ; h \in \mathcal{B}\right\}$ for $\frac{\mathbb{C}\{x, y\}}{J(f)}$.

Notice that by the previous theorem we get

$$
\nu_{f}(J(f))-\left(\mu_{f}-1\right)=\Gamma_{f} \backslash\{0\}
$$

In [Z3], Zariski obtains (11) considering the minimal polynomial $f_{k}(x, y) \in \mathbb{C}\{x\}[y]$ of the element $\sum_{m \leq i<\beta_{k}} a_{i} x^{\frac{i}{n_{0} \cdot n_{1} \cdots \cdot n_{k}}} \in \mathbb{C}(x)$ and he shows that $\nu_{f}\left(f_{k}\right)=v_{k+1}$. This is a particular case of semiroots associated to $f$.

Definition 2.3. Given $k \in\{0, \ldots, g\}$ a monic polynomial $f_{k} \in \mathbb{C}\{x\}[y]$ of degree $n_{0} \cdot n_{1} \cdot \ldots \cdot n_{k}=$ $\frac{n}{e_{k}}$ is called a k-semiroot of $f$ if $\nu_{f}\left(f_{k}\right)=v_{k+1}$ where $v_{g+1}=\infty$.

Any semiroot $f_{k}$ is irreducible, the associated curve $C_{k}:=C_{f_{k}}$ has characteristic sequence $\frac{\beta_{0}}{e_{k}}, \ldots, \frac{\beta_{k}}{e_{k}}$ and it admits semigroup $\Gamma_{k}=\left\langle\frac{v_{0}}{e_{k}}, \ldots, \frac{v_{k}}{e_{k}}\right\rangle$ (see Theorem 5.1 in Pop).

If $C_{f}$ admits a Puiseux parametrization $\left(t^{\beta_{0}}, \varphi(t)\right)$ and $f_{k}$ is a $k$-semiroot of $f$ then $C_{k}$ admits a Puiseux parametrization $\left(t^{\frac{\beta_{0}}{e_{k}}}, \psi(t)\right)$ with $j^{\beta_{k}}(\varphi(t))=j^{\beta_{k}}\left(\psi\left(t^{e_{k}}\right)\right)$ where $j^{r}(h)$ denotes the $r$ jet of $h$ (see Pop, Corollary 5.3). In addition, if $f_{k}$ is a $k$-semiroot of $f$ and $f_{j}$ is a $j$-semiroot of $f_{k}$ then $f_{j}$ is a $j$-semiroot of $f$.

A set $\left\{f_{k} ; f_{k}\right.$ is a $k$-semiroot of $f$ with $\left.0 \leq k \leq g\right\}$ is called a complete system of semiroots of $f$. A complete system of semiroots can be used to express any $h \in \mathbb{C}\{x\}[y]$ in a highlighted way as follows. 
Lemma 2.4 ( $(\overline{\text { Pop }}$, Corollary 5.4). Any element $h \in \mathbb{C}\{x\}[y]$ can be uniquely expressed as

$$
h=\sum_{\substack{\text { finite } \\ \alpha=\left(\alpha_{0}, \ldots, \alpha_{g}\right) \in \mathbb{N}^{g}+1}} b_{\alpha} f_{0}^{\alpha_{0}} \cdots f_{g-1}^{\alpha_{g-1}} f_{g}^{\alpha_{g}}
$$

with $0 \leq \alpha_{g} \leq\left[\frac{g r(h)}{g r(f)}\right], 0 \leq \alpha_{k}<n_{k+1}$ for $0 \leq k<g$ and $b_{\alpha} \in \mathbb{C}\{x\}$. Moreover:

1. $\operatorname{deg}_{y}\left(f_{0}^{\alpha_{0}} \cdots f_{g}^{\alpha_{g}}\right) \neq \operatorname{deg}_{y}\left(f_{0}^{\gamma_{0}} \cdots f_{g}^{\gamma_{g}}\right)$ if and only if $\alpha \neq \gamma$.

2. $\nu_{f}\left(b_{\alpha} f_{0}^{\alpha_{0}} \cdots f_{g-1}^{\alpha_{g-1}}\right) \neq \nu_{f}\left(b_{\gamma} f_{0}^{\gamma_{0}} \cdots f_{g-1}^{\gamma_{g-1}}\right)$ for $\left(\alpha_{0}, \ldots, \alpha_{g-1}\right) \neq\left(\gamma_{0}, \ldots, \gamma_{g-1}\right)$.

Remark 2.5. Given $h \in \mathbb{C}\{x\}[y]$ as expressed in the previous lemma with $\alpha_{g}=0$ for every $\alpha \in \mathbb{N}^{g+1}$ and $\nu_{f}(h)=\nu_{f}\left(b_{\alpha} f_{0}^{\alpha_{0}} \cdots f_{g-1}^{\alpha_{g-1}}\right)=\sum_{i=1}^{g} \alpha_{i-1} v_{i}$. If $\alpha_{i-1}=n_{i}-1$ for $1 \leq i \leq g$ then

$$
\operatorname{deg}_{y}(h)=\operatorname{deg}_{y}\left(f_{0}^{\alpha_{0}} \cdots f_{g-1}^{\alpha_{g-1}}\right)=\sum_{i=1}^{g}\left(n_{i}-1\right) n_{0} \cdot n_{1} \cdot \ldots \cdot n_{i-1}=n-1 .
$$

In what follows for $0 \leq k \leq g$ we consider the multiplicative group $G_{k}=\left\{\eta \in \mathbb{C} ; \eta^{e_{k}}=1\right\}$. Remark that $\{1\}=G_{g} \subset \cdots \subset G_{1} \subset G_{0}$ and $n_{i}=\sharp \frac{G_{i-1}}{G_{i}}$ for all $1 \leq i \leq g$.

The next result relates $\nu_{f}(h)$ with $\nu_{k}(h):=\nu_{f_{k}}(h)$.

Proposition 2.6. Let $f_{j}$ be a j-semiroot of $f_{k}$ and $f_{k}$ a $k$-semiroot of $f=f_{g} \in \mathbb{C}\{x\}[y]$ with $\left(t^{\frac{v_{0}}{e_{j}}}, \phi(t)\right),\left(t^{\frac{v_{0}}{e_{k}}}, \psi(t)\right)$ and $\left(t^{v_{0}}, \varphi(t)\right)$ their respective parametrizations. If $f_{j}\left(t^{\frac{v_{0}}{e_{k}}}, \psi(t)\right)=$ $a t^{\frac{v_{j}}{e_{k}}}+\left(\right.$ h.o.t.), then $f_{j}\left(t^{v_{0}}, \varphi(t)\right)=a t^{v_{j}}+$ (h.o.t.).

In particular, for any $h \in \mathbb{C}\{x\}[y]$ with $\operatorname{deg}_{y}(h)<\operatorname{deg}_{y}\left(f_{k}\right)$ and $h\left(t^{\frac{v_{0}}{e_{k}}}, \psi(t)\right)=b t^{\nu_{k}(h)}+$ (h.o.t.), we get $h\left(t^{v_{0}}, \varphi(t)\right)=b t^{\nu_{f}(h)}+$ (h.o.t.) with $\nu_{f}(h)=e_{k} \nu_{k}(h)$.

Proof: Considering $\varphi(t)=\sum_{i \geq v_{0}} a_{i} t^{i}$, according with Corollary 5.3, Proposition 6.5 in Pop, sections 6.2 and 8.1 in $[\mathrm{H}]$, for every $0<i<j<k \leq g$ we obtain:

$$
\psi_{k}(t)-\phi\left(\zeta t^{\frac{e_{j}}{e_{k}}}\right)= \begin{cases}a_{\beta_{i}}\left(1-\zeta^{\frac{\beta_{i}}{e_{j}}}\right) t^{\frac{\beta_{i}}{e_{k}}}+(\text { h.o.t.) } & \text { if } \zeta \in G_{i-1} \backslash G_{i} ; \\ a_{\beta_{j}} t^{\frac{\beta_{j}}{e_{k}}}+(\text { h.o.t. }) & \text { if } \zeta \in G_{j},\end{cases}
$$

with $\zeta^{\frac{\beta_{i}}{e_{j}}} \neq 1$ if $\zeta \in G_{i-1} \backslash G_{i}$.

As $f_{j}(x, y)=\prod_{\bar{\zeta} \in \frac{G_{0}}{G_{j}}}\left(y-\phi\left(\zeta x^{\frac{e_{j}}{v_{0}}}\right)\right)$ we have $f_{j}\left(t^{\frac{v_{0}}{e_{k}}}, \psi(t)\right)=a t^{\frac{v_{j}}{e_{k}}}+$ (h.o.t.), with $a \neq 0$ and taking $k=g$ we get $f_{j}\left(t^{v_{0}}, \varphi(t)\right)=a t^{v_{j}}+$ (h.o.t.).

Moreover, given $h \in \mathbb{C}\{x\}[y]$ with $\operatorname{gr}(h)<\operatorname{gr}\left(f_{k}\right)$ by Lemma 2.4 we can express $h=$ $\sum_{\alpha \in \mathbb{N}^{k}} b_{\alpha} f_{0}^{\alpha_{0}} \cdots f_{k-1}^{\alpha_{k-1}}$ with $0 \leq \alpha_{j}<n_{j+1}$ for all $0 \leq j<k, b_{\alpha} \in \mathbb{C}\{x\}$ and there exists a unique $\gamma \in \mathbb{N}^{k}$ such that

$$
\nu_{f}(h)=\nu_{f}\left(b_{\gamma} f_{0}^{\gamma_{0}} \cdots f_{k-1}^{\gamma_{k-1}}\right)=\sum_{j=1}^{k-1} \gamma_{j-1} v_{j}+v_{0} \cdot \operatorname{ord}_{x}\left(b_{\alpha}\right)=e_{k} \nu_{f_{k}}\left(b_{\gamma} f_{0}^{\gamma_{0}} \cdots f_{k-1}^{\gamma_{k-1}}\right)=e_{k} \nu_{f_{k}}(h) .
$$


In addition, as $f_{j}\left(t^{\frac{v_{0}}{e_{k}}}, \psi(t)\right)=a t^{\frac{v_{j}}{e_{k}}}+$ (h.o.t.) and $f_{j}\left(t^{v_{0}}, \varphi(t)\right)=a t^{v_{j}}+$ (h.o.t.) for all $0 \leq j<k$, if $h\left(t^{\frac{v_{0}}{e_{k}}}, \psi(t)\right)=b t^{\nu_{k}(h)}+$ (h.o.t.), then $h\left(t^{v_{0}}, \varphi(t)\right)=b t^{\nu_{f}(h)}+$ (h.o.t.).

Notice that $\Gamma_{f}=e_{k} \Gamma_{k}+\left\langle v_{k+1}, \ldots, v_{g}\right\rangle$ and

$$
n_{j}=\frac{e_{j-1}}{e_{j}}=\frac{\operatorname{gcd}\left(\beta_{0}, \ldots, \beta_{j-1}\right)}{\operatorname{gcd}\left(\beta_{0}, \ldots, \beta_{j}\right)}=\frac{\operatorname{gcd}\left(\frac{\beta_{0}}{e_{k}}, \ldots, \frac{\beta_{j-1}}{e_{k}}\right)}{\operatorname{gcd}\left(\frac{\beta_{0}}{e_{k}}, \ldots, \frac{\beta_{j}}{e_{k}}\right)} \text { for every } j=1, \ldots, k .
$$

In this way, if $\mu_{k}$ denotes the Milnor number of $C_{k}$ we have

$$
\mu_{f}-1=\sum_{j=1}^{g}\left(n_{j}-1\right) v_{j}-v_{0}=e_{k}\left(\mu_{k}-1\right)+\sum_{j=k+1}^{g}\left(n_{j}-1\right) v_{j} .
$$

The above remarks indicate how some topological data of a branch and of their semiroots are related. More delicate question is: How we can relate analytical invariants of $C_{f}$ and $C_{k}$ ? Investigating this question is one of the aim of this work.

In the next section we will present results about some analytical invariants of a plane branch associated with differential forms that play an important role in the main results of this work.

\section{Differential forms on $C_{f}$}

Let $\Omega^{1}=\mathbb{C}\{x, y\} d x+\mathbb{C}\{x, y\} d y$ be the $\mathbb{C}\{x, y\}$-module of holomorphic forms on $\mathbb{C}^{2}$.

Given $f \in \mathbb{C}\{x, y\}$ we denote $d f=f_{x} d x+f_{y} d y$ and $\mathcal{F}(f)=f \cdot \Omega^{1}+\mathbb{C}\{x, y\} \cdot d f$. The Kähler differential module of the branch $C_{f}$ is the $\mathcal{O}_{f}$-module $\Omega_{f}:=\Omega_{\mathcal{O}_{f} / \mathbb{C}}=\frac{\Omega^{1}}{\mathcal{F}(f)}$. If $C_{f}$ is singular then $\Omega_{f}$ has a non trivial torsion submodule $\mathcal{T}_{f}=\left\{\omega \in \Omega_{f} ; h \omega=0\right.$ for some $\left.0 \neq h \in \mathcal{O}_{f}\right\}$ and $\ell\left(\mathcal{T}_{f}\right)=\tau_{f}=\operatorname{dim}_{\mathbb{C}} \frac{\mathbb{C}\{x, y\}}{\left\langle f, f_{x}, f_{y}\right\rangle}$ (see [Z2] for instance).

Considering a parametrization $(x(t), y(t)) \in \mathbb{C}\{t\} \times \mathbb{C}\{t\}$ of $C_{f}$ and the identification $\mathcal{O}_{f} \approx$ $\mathbb{C}\{x(t), y(t)\}$ we define the $\mathcal{O}_{f}$-homomorphism

$$
\begin{aligned}
& \Upsilon: \quad \Omega_{f} \quad \rightarrow \quad \mathbb{C}\{t\} \approx \overline{\mathcal{O}}_{f} \\
& A(x, y) d x+B(x, y) d y \mapsto t \cdot\left(A(x(t), y(t)) \cdot x^{\prime}(t)+B(x(t), y(t)) \cdot y^{\prime}(t)\right),
\end{aligned}
$$

and $\operatorname{ker}(\Upsilon)=\mathcal{T}_{f}$.

The set $\Lambda_{f}=\left\{\nu_{f}(\omega):=\operatorname{ord}_{t}(\Upsilon(\omega)) ; \overline{0} \neq \bar{\omega} \in \frac{\Omega_{f}}{\mathcal{T}_{f}}\right\}$ is an analytical invariant of $C_{f}$ and $\Gamma_{f} \backslash\{0\}=\left\{\nu_{f}(d h) ; 0 \neq h \in \mathcal{O}_{f} \cap\langle t\rangle\right\} \subseteq \Lambda_{f}$. The set $\Lambda_{f}$ has a prominent role in many works (see [De] and [HH3] for instance) and it is related with the Tjurina number $\tau_{f}$ of $C_{f}$ by the relation $\tau_{f}=\mu_{f}-\sharp\left(\Lambda_{f} \backslash \Gamma_{f}\right)$. In [HH2] we found an algorithm to compute the set $\Lambda_{f}$ for any irreducible curve plane or not and all possible set of values of differentials for plane curves with a fixed semigroup.

According to Saito (see $\left[\underline{S}\right.$ ) an element $W \in \frac{1}{f} \Omega^{1}$ is a logarithmic form along $C_{f}$ if there exist $\varpi \in \Omega^{1}, P, Q \in \mathbb{C}\{x, y\}, Q \notin\langle f\rangle$ such that $Q W=\frac{P d f+f \varpi}{f}$. Saito denotes $\Omega\left(\log C_{f}\right)=$ 
$\left\{W ; W\right.$ is a logarithmic form along $\left.C_{f}\right\}$ and motivated by this, in the sequel, we will consider the $\mathbb{C}\{x, y\}$-module $f \cdot \Omega\left(\log C_{f}\right)=\left\{f \cdot W ; W \in \Omega\left(\log C_{f}\right)\right\} \subset \Omega^{1}$. It follows that $\mathcal{T}_{f}=\frac{f \cdot \Omega\left(\log C_{f}\right)}{\mathcal{F}(f)}$.

In section 2 of [S], Saito introduce the residue of a logarithmic form that can be defined considering $f \cdot \Omega\left(\log C_{f}\right)$. More precisely, given $\omega \in f \cdot \Omega\left(\log C_{f}\right)$ with $Q \omega=P d f+f \varpi$ we call $\operatorname{res}(\omega)=\frac{\bar{P}}{\bar{Q}} \in \operatorname{Frac}\left(\mathcal{O}_{f}\right)$ the residue of $\omega$ where $\operatorname{Frac}\left(\mathcal{O}_{f}\right)$ is the field of fraction of $\mathcal{O}_{f}$. We have that $\operatorname{res}(\omega)$ is well defined and $\mathcal{R}_{f}:=\left\{\operatorname{res}(\omega) ; \omega \in f \cdot \Omega\left(\log C_{f}\right)\right\} \supseteq \overline{\mathcal{O}}_{f}$.

Pol (see Corollary 3.32, $[\mathrm{Pol}]$ ) shows that $\Lambda_{f}$ determines and it is determined by the set $\Delta_{f}:=\left\{\nu_{f}(P)-\nu_{f}(Q) ; \overline{\bar{Q}} \in \mathcal{R}_{f}\right\}$. More precisely, she proves that $\lambda \in \Lambda_{f}$ if and only if $-\lambda \notin \Delta_{f}$.

Remark 3.1. Given $\omega \in \Omega^{1}$ we denote $P_{f}(\omega):=\frac{\omega \wedge d f}{d x \wedge d y} \in J(f)$. In this way, we have that $f \cdot \Omega\left(\log C_{f}\right)=\left\{\omega \in \Omega^{1} ; P_{f}(\omega) \in\langle f\rangle\right\}$, that is, $\omega=A d x-B d y \in f \cdot \Omega\left(\log C_{f}\right)$ if and only if $A f_{y}+B f_{x}=P_{f}(\omega)=M f$ for some $M \in \mathbb{C}\{x, y\}$, or equivalently, $\frac{P_{f}(\omega)}{f} \in(J(f):\langle f\rangle)$. In particular, we have

$$
f_{x} \omega=A d f-f M d y \quad \text { and } \quad f_{y} \omega=-B d f+f M d x
$$

that is, res $(\omega)=\frac{A}{f_{x}}=\frac{B}{f_{y}}$. In addition, $\omega \in \mathcal{F}(f)$ if and only if $\frac{P_{f}(\omega)}{f} \in J(f) \subseteq(J(f):\langle f\rangle)$.

The rest of this section is devoted to show that $\nu_{f}((J(f):\langle f\rangle))$ and $\Delta_{f}$ (consequently, $\left.\Lambda_{f}\right)$ are mutually determined and to revisit some results in [BMT] about equations $A f_{y}+B f_{x}=C$.

As in the Section 2 we will assume that $f \in \mathbb{C}\{x\}[y]$ is an irreducible Weierstrass polynomial with $v_{1}=\nu_{f}(y)>\nu_{f}(x)=v_{0}=n=\operatorname{deg}_{y}(f)=\operatorname{mult}(f)>1$ and $v_{0} \nmid v_{1}$. In this way, we have $\nu_{f}\left(f_{x}\right)=\mu_{f}-1+v_{1}, \nu_{f}\left(f_{y}\right)=\mu_{f}-1+v_{0}=\sum_{i=1}^{g}\left(e_{i-1}-e_{i}\right) \beta_{i}$ (see Corollaries 7.15 and 7.16, [H]) and by (4)

$$
\Delta_{f}=\left\{\nu_{f}(B)-\left(\mu_{f}-1+v_{0}\right) ; \omega=A d x+B d y \in f \cdot \Omega\left(\log C_{f}\right)\right\} .
$$

Remark 3.2. With the previous notation we have $\nu_{f}(J(f))=\left\{\nu_{f}\left(\frac{P_{f}(\omega)}{f}\right) ; \omega \in \mathcal{F}(f)\right\}$ and

$$
\left\{\nu_{f}(\operatorname{res}(\omega)) \neq 0 ; \omega \in \mathcal{F}(f)\right\}=\Gamma_{f} \backslash\{0\}=\nu_{f}(J(f))-\left(\mu_{f}-1\right) .
$$

In fact, as $\mathcal{F}(f)=f \cdot \Omega^{1}+\mathbb{C}\{x, y\} \cdot d f$ it follows that $\left\{\frac{P_{f}(\omega)}{f} ; \omega \in \mathcal{F}(f)\right\}=J(f)$ and $\mathbb{C}\{x, y\}=$ $\{\operatorname{res}(\omega) ; \omega \in \mathcal{F}(f)\}$. We get the conclusion by (3).

For the next result we introduce the following $\mathbb{C}$-vector spaces:

$$
\begin{array}{cc}
\mathcal{P}_{s}=\left\{h \in \mathbb{C}\{x\}[y] ; \operatorname{deg}_{y}(h)<s\right\} ; & \mathcal{E}(f)=\mathcal{P}_{n} d x+\mathcal{P}_{n-1} d y \\
\mathcal{G}(f)=\mathbb{C}\{x, y\} d f+\mathbb{C}\{x, y\} f d x ; & \mathcal{H}(f)=\mathbb{C}\{x\} d f+f \Omega^{1} .
\end{array}
$$

Lemma 3.3. With the above notation we have $\mathcal{E}(f) \oplus \mathcal{G}(f)=\Omega^{1}=\mathcal{E}(f) \oplus \mathcal{H}(f)$.

Proof: Given $\omega=A d x+B d y \in \Omega^{1}$, by the Weierstrass division theorem we can write $B=Q f_{y}+B_{1}$ with $B_{1} \in \mathcal{P}_{n-1}$ and $A-Q f_{x}=P f+A_{1}$ with $A_{1} \in \mathcal{P}_{n}$. So,

$$
\omega=\left(A_{1} d x+B_{1} d y\right)+(Q d f+P f d x) \in \mathcal{E}(f)+\mathcal{G}(f) .
$$


If $\omega \in \mathcal{E}(f) \cap \mathcal{G}(f)$ there exist $A_{1} \in \mathcal{P}_{n}, B_{1} \in \mathcal{P}_{n-1}, P, Q \in \mathbb{C}\{x, y\}$ such that $A_{1} d x+B_{1} d y=$ $\omega=Q d f+P f d x$, that is, $A_{1}=Q f_{x}+P f$ and $B_{1}=Q f_{y}$. As $\operatorname{deg}_{y}\left(f_{y}\right)=\operatorname{deg}_{y}(f)-1=n-1$ it follows that $Q=P=0$, that is, $\omega=0$. Hence, $\Omega^{1}=\mathcal{E}(f) \oplus \mathcal{G}(f)$.

For the other equality we write $B=Q_{1} f+B_{1}, B_{1}=Q_{0} f_{y}+B_{0}$ and $A-Q_{0} f_{x}=P_{1} f+A_{0}$ with $B_{1}, A_{0} \in \mathcal{P}_{n}, B_{0} \in \mathcal{P}_{n-1}$ and $Q_{0} \in \mathcal{P}_{1}=\mathbb{C}\{x\}$. So,

$$
\omega=A d x+B d y=\left(A_{0} d x+B_{0} d y\right)+\left(Q_{0} d f+f\left(P_{1} d x+Q_{1} d y\right)\right) \in \mathcal{E}(f)+\mathcal{H}(f)
$$

and similarly to the previous case we conclude $\mathcal{E}(f) \oplus \mathcal{H}(f)$.

In $[\mathrm{BHH}$ the authors call the expression (5) is called the Weierstrass form of $\omega$ and it was considered to study some properties of the foliation associated to $\omega$.

Notice that if $\omega=Q d f+P f d x \in \mathcal{G}(f) \subset \mathcal{F}(f) \subset f \cdot \Omega\left(\log C_{f}\right)$ then $\nu_{f}(\operatorname{res}(\omega))=\nu_{f}(Q)$ does not have any relation with $\nu_{f}\left(\frac{P_{f}(\omega)}{f}\right)=\nu_{f}\left(P f_{y}\right)$. On the other hand, we will show that for $\omega \in \mathcal{E}(f) \cap f \cdot \Omega\left(\log C_{f}\right)$ the values $\nu_{f}(\operatorname{res}(\omega))$ and $\nu_{f}\left(\frac{P_{f}(\omega)}{f}\right)$ are mutually determined.

In the sequel we follow the ideas of [BMT].

We denote the roots of $f \in \mathbb{C}\{x\}[y]$ by $\left\{\varphi_{i}:=\varphi\left(\zeta^{j} x^{\frac{1}{n}}\right) ; 1 \leq j \leq n\right\} \subset \mathbb{C}\left\{x^{\frac{1}{n}}\right\}$ where $\zeta$ is an $n$-th primitive root of the unity, $\mathbb{K}:=\operatorname{Frac}\left(\mathbb{C}\left\{x^{\frac{1}{n}}\right\}\right)$ and $\mathcal{P}_{s}^{\mathbb{K}}:=\left\{h \in \mathbb{K}[y] ; \operatorname{deg}_{y}(h)<s\right\}$.

Let us consider the $\mathbb{K}$-basis $\mathbb{B}=\left\{\Phi_{i}:=\frac{f}{y-\varphi_{i}}=\prod_{\substack{j=1 \\ j \neq i}}^{n}\left(y-\varphi_{j}\right) ; i=1, \ldots, n\right\}$ for $\mathcal{P}_{n}^{\mathbb{K}}$. Notice that $\Phi_{i}\left(\varphi_{k}\right)=\prod_{\substack{j=1 \\ j \neq i}}^{n}\left(\varphi_{k}-\varphi_{j}\right) ; i=1, \ldots, n$,

$$
f_{y}\left(\varphi_{k}\right)=\Phi_{k}\left(\varphi_{k}\right) \quad \text { and } \quad f_{x}\left(\varphi_{k}\right)=-\varphi_{k}^{\prime} \Phi_{k}\left(\varphi_{k}\right)=-\varphi_{k}^{\prime} f_{y}\left(\varphi_{k}\right) .
$$

Moreover, given an element $h \in \mathcal{P}_{n}^{\mathbb{K}}$ we can express it using the basis $\mathbb{B}$ as $h=\sum_{i=1}^{n} h_{i} \Phi_{i}$ with $h_{i}=\frac{h\left(\varphi_{i}\right)}{\Phi_{i}\left(\varphi_{i}\right)} \in \mathbb{K}$.

Now, let us consider $\omega=A d x-B d y \in \mathcal{E}(f) \cap f \cdot \Omega\left(\log C_{f}\right)$ with

$$
P_{f}(\omega)=B f_{x}+A f_{y}=M f .
$$

As $A \in \mathcal{P}_{n}$ and $B \in \mathcal{P}_{n-1}$ we get $M \in \mathcal{P}_{n-1} \subset \mathcal{P}_{n-1}^{\mathbb{K}}$.

The next result relates $M$ with $B$ by means the $\mathbb{K}$-basis $\mathbb{B}$.

Lemma 3.4 (Lemma 2.2, [BMT] $)$. Given $M=\sum_{i=1}^{n} M_{i} \Phi_{i}, B=\sum_{i=1}^{n} B_{i} \Phi_{i} \in \mathcal{P}_{n-1}^{\mathbb{K}}$ and $A=$ $\sum_{i=1}^{n} A_{i} \Phi_{i} \in \mathcal{P}_{n}^{\mathbb{K}}$ satisfying (6) we have $A_{i}=B_{i} \varphi_{i}^{\prime}$ and

$$
M_{i}=\left(\sum_{\substack{j=1 \\ j \neq i}}^{n}\left(\frac{\varphi_{i}^{\prime}-\varphi_{j}^{\prime}}{\varphi_{i}-\varphi_{j}}\right)\right) \cdot B_{i}-\sum_{\substack{j=1 \\ j \neq i}}^{n}\left(\left(\frac{\varphi_{i}^{\prime}-\varphi_{j}^{\prime}}{\varphi_{i}-\varphi_{j}}\right) \cdot B_{j}\right), \quad \text { for every } i=1, \ldots, n .
$$

As $\nu_{f}(M)=\operatorname{ord}_{t}\left(M\left(t^{n}, \varphi_{n}(t)\right)\right)$ and $\nu_{f}(B)=\operatorname{ord}_{t}\left(B\left(t^{n}, \varphi_{i}(t)\right)\right)$ there exist $b, c, d, u_{j} \in \mathbb{C} \backslash\{0\}$ such that

$$
\begin{array}{ll}
\left.B\left(x, \varphi_{i}\right)=b . \zeta^{i \nu_{f}(B)} x^{\frac{\nu_{f}(B)}{n}}+\text { (h.o.t. }\right), & \Phi_{i}\left(\varphi_{i}\right)=f_{y}\left(x, \varphi_{i}\right)=c . \zeta^{i \nu_{f}\left(f_{y}\right)} x^{\frac{\nu_{f}\left(f_{y}\right)}{n}}+(\text { h.o.t }), \\
\left.M\left(x, \varphi_{n}\right)=d . x^{\frac{\nu_{f}(M)}{n}}+\text { (h.o.t. }\right), & \varphi_{n}-\varphi_{j}=u_{j} x^{\frac{m_{j}}{n}}+(\text { h.o.t. })(j \neq n),
\end{array}
$$


where $\zeta$ is an $n$-th primitive root of the unity and $m_{j}=\beta_{k}$ if $\zeta^{j} \in G_{k-1} \backslash G_{k}$. So, we get $-\left(\frac{\varphi_{n}^{\prime}-\varphi_{j}^{\prime}}{\varphi_{n}-\varphi_{j}}\right)=-\frac{m_{j}}{n x}+$ (h.o.t.).

Considering $i=n$ in (17) and expanding the expressions we obtain

$$
d \cdot x^{\frac{\nu_{f}(M)-\nu_{f}\left(f_{y}\right)}{n}}+(\text { h.o.t. })=\sum_{i=1}^{n-1} m_{i}\left(1-\zeta^{i\left(\nu_{f}(B)-\nu_{f}\left(f_{y}\right)\right)}\right) \cdot \frac{b}{n} \cdot x^{\frac{\nu_{f}(B)-\nu_{f}\left(f_{y}\right)-n}{n}}+\text { (h.o.t.). }
$$

Denote $S:=\sum_{i=1}^{n-1} m_{i}\left(1-\zeta^{i\left(\nu_{f}(B)-\nu_{f}\left(f_{y}\right)\right)}\right)$. As, $m_{i}=\beta_{k}$ for $\eta=\zeta^{i} \in G_{k-1} \backslash G_{k}$ we get

$$
S=\sum_{i=1}^{g}\left(\sum_{\eta \in G_{i-1} \backslash G_{i}}\left(1-\eta^{\nu_{f}(B)-\nu_{f}\left(f_{y}\right)}\right)\right) \beta_{i} \text {. }
$$

The next two lemmas allow us to conclude that $S \neq 0$, consequently we obtain $\nu_{f}(M)=$ $\nu_{f}(B)-n$ and $d=\frac{S \cdot b}{n}$.

Lemma 3.5. If $B \in \mathcal{P}_{n-1}$ with $\nu_{f}(B)=\sum_{i=0}^{g} s_{i} v_{i}, 0 \leq s_{i}<n_{i}$ and $s_{0} \geq 0$ then there exists $k \in G_{0}$ such that $e_{k} \nmid \alpha:=\sum_{i=1}^{g}\left(n_{i}-1-s_{i}\right) v_{i}$.

Proof: Let us consider a complete system of semiroots $\left\{f_{0}, \ldots, f_{g}\right\}$ of $f$. As $\operatorname{deg}_{y}(B)<n-1$, according Lemma 2.4 we can write

$$
B=\sum_{\substack{\text { finite } \\ r \in \mathbb{N} g+1}} a_{r} f_{0}^{r_{1}} \cdots f_{g-1}^{r_{g}} \text { with } \nu_{f}(B)=\nu_{f}\left(a_{s} f_{0}^{s_{1}} \cdots f_{g-1}^{s_{g}}\right)=\sum_{i=0}^{g} s_{i} v_{i} .
$$

Let us suppose that $e_{k} \mid \alpha$ for every $k \in G_{0} \backslash\{1\}$. If there exists $k=\max \left\{i>0 ; s_{i}<n_{i}-1\right\}$ then, by (11) and (2), we have $\left(n_{i}-1-s_{i}\right) v_{i}>n_{i-1} v_{i-1}>e_{i-1}\left(\mu_{i-1}-1\right)$. But this implies $\left(n_{i}-\right.$ $\left.1-s_{i}\right) v_{i} \in\left\langle v_{0}, \ldots, v_{i-1}\right\rangle$ that is a contradiction because $n_{i}=\min \left\{j>0 ; j . v_{i} \in\left\langle v_{0}, \ldots, v_{i-1}\right\rangle\right\}$. So, if $e_{k} \mid \alpha$ for every $k \in G_{0} \backslash\{1\}$ then $s_{k}=n_{k}-1$ for every $1 \leq k \leq g$. By Remark 2.5] we would have $\operatorname{deg}_{y}(B)=n-1$ that is an absurd.

Lemma 3.6. Given $\alpha \in \mathbb{Z}$ and $k=\max \left\{i \in G_{0} ; e_{i} \nmid \alpha\right\}$ we have

$$
\sum_{\eta \in G_{j} \backslash G_{j+1}} \eta^{\alpha}= \begin{cases}e_{j}-e_{j+1} & \text { if } k+1 \leq j<g \\ -e_{j+1} & \text { if } j=k \\ 0 & \text { if } 0 \leq j<k .\end{cases}
$$

Proof: $\quad$ As $\sharp G_{k}=e_{k}$ we have that $\sum_{\eta \in G_{k}} \eta^{\alpha}=\left\{\begin{array}{lll}0 & \text { if } & e_{k} \nmid \alpha \\ e_{k} & \text { if } & e_{k} \mid \alpha .\end{array}\right.$

Therefore, the result follows considering $\sum_{\eta \in G_{j}} \eta^{\alpha}=\sum_{\eta \in G_{j} \backslash G_{j+1}} \eta^{\alpha}+\sum_{\eta \in G_{j+1}} \eta^{\alpha}$ and recalling that $e_{j} \mid e_{k}$ for $j \leq k$.

As a consequence of the previous two lemmas we obtain the following result. 
Proposition 3.7. If $\omega=A d x-B d y \in \mathcal{E}(f) \cap f \cdot \Omega\left(\log C_{f}\right)$ with $P_{f}(\omega)=B f_{x}+A f_{y}=M f$ then

$$
M\left(t^{n}, \varphi_{n}(t)\right)=\frac{e_{k} v_{k+1} b}{n} t^{\nu_{f}(M)}+(\text { h.o.t. }), \quad B\left(t^{n}, \varphi_{n}(t)\right)=b t^{\nu_{f}(B)}+(\text { h.o.t. })
$$

and $\nu_{f}(B)=\nu_{f}(M)+v_{0}$, where $k=\max \left\{i \in G_{0} ; e_{i} \nmid \nu_{f}(B)-\nu_{f}\left(f_{y}\right)\right\}$.

ProOF: Remark that Lemma 3.5 give us the existence of $k=\max \left\{i \in G_{0} ; e_{i} \nmid \nu_{f}(B)-\nu_{f}\left(f_{y}\right)\right\}$. By (9) it is sufficient to show that in (10) we have $S=\sum_{i=1}^{g}\left(\sum_{\eta \in G_{i-1} \backslash G_{i}}\left(1-\eta^{\nu_{f}(B)-\nu_{f}\left(f_{y}\right)}\right)\right) \beta_{i} \neq$ 0 . But this is a consequence of Lemma 3.6 and (1) because

$$
S=\sum_{i=1}^{g}\left(e_{i-1}-e_{i}\right) \beta_{i}-\left(-e_{k+1} \beta_{k+1}+\sum_{j=k+2}^{g}\left(e_{j-1}-e_{j}\right) \beta_{j}\right)=\sum_{i=1}^{k}\left(e_{i-1}-e_{i}\right) \beta_{i}+e_{k} \beta_{k+1}=e_{k} v_{k+1} \neq 0 .
$$

The next result shows that $\Delta_{f}$ and $\nu_{f}(J(f): f)$ are mutually determined.

Theorem 3.8. Let $f \in \mathbb{C}\{x\}[y]$ be an irreducible Weierstrass polynomial with $\operatorname{deg}_{y}(f)=v_{0}=n$ and $\Gamma_{f}=\left\langle v_{0}, v_{1}, \ldots, v_{g}\right\rangle$, then $\left\{\delta \neq 0 ;-\delta \notin \Lambda_{f}\right\}=\Delta_{f} \backslash\{0\}=\nu_{f}(J(f): f)-\left(\mu_{f}-1\right)$.

Proof: By Pol (see Corollary 3.32, [Pol]) it follows that $\left\{\delta ;-\delta \notin \Lambda_{f}\right\}=\Delta_{f}$ and by Remark (3.2) we have that $\Gamma_{f} \backslash\{0\} \subseteq \Delta_{f} \cap\left(\nu_{f}(J(f): f)-\left(\mu_{f}-1\right)\right)$.

Given $\alpha \in \Delta_{f} \backslash \Gamma_{f}$ there exists $\omega=A d x+B d y \in f \cdot \Omega\left(\log C_{f}\right)$ with $\nu_{f}(\operatorname{res}(\omega))=\nu_{f}\left(\frac{B}{f_{y}}\right)=\alpha$ and, according to Lemma 3.3, we can write

$$
\omega=A d x+B d y=A_{0} d x+B_{0} d y+R d f+(P d x+Q d y) f \in \mathcal{E}(f) \oplus \mathcal{H}(f),
$$

with $A_{0} d x+B_{0} d y \in \mathcal{E}(f) \cap f \cdot \Omega\left(\log C_{f}\right)$ and $R \in \mathbb{C}\{x\}$. In particular, $\alpha+\mu_{f}-1+v_{0}=\nu_{f}(B)=$ $\nu_{f}\left(B_{0}+R f_{y}+Q f\right)=\nu_{f}\left(B_{0}+R f_{y}\right)$ and $\nu_{f}(R)=s_{0} v_{0}$.

If $\nu_{f}\left(R f_{y}\right)<\nu_{f}\left(B_{0}\right)$ we should have $\alpha+\mu_{f}-1+v_{0}=\nu_{f}\left(R f_{y}\right)=s_{0} v_{0}+\mu_{f}-1+v_{0}$, i.e., $\alpha=s_{0} v_{0} \in \Gamma_{f}$ that is an absurd.

If $\nu_{f}\left(B_{0}\right)=\nu_{f}\left(R f_{y}\right)$, then $\nu_{f}\left(B_{0}\right)=s_{0} v_{0}+\sum_{i=1}^{g}\left(n_{i}-1\right) v_{i}$. But, in this way, Lemma 2.5 gives us $\operatorname{deg}_{y}\left(B_{0}\right) \geq n-1$ and we get a contradiction.

In this way $\nu_{f}\left(R f_{y}\right)>\nu_{f}\left(B_{0}\right)=\nu_{f}(B)=\alpha+\mu_{f}-1+v_{0}$ and taking $\omega_{0}=A_{0} d x+$ $B_{0} d y \in \mathcal{E}(f) \cap f \cdot \Omega\left(\log C_{f}\right)$ with $P_{f}\left(\omega_{f}\right)=M_{0} f$ we have $M_{0} \in(J(f): f)$, by Proposition 3.7, $\alpha+\mu_{f}-1=\nu_{f}(B)-v_{0}=\nu_{f}\left(B_{0}\right)-v_{0}=\nu_{f}\left(M_{0}\right) \in \nu_{f}(J(f): f)$.

Reciprocally, consider $\alpha+\mu_{f}-1=\nu_{f}(M) \in \nu_{f}(J(f): f)$ with $\alpha \notin \Gamma_{f}$.

According to Saito, we have $\overline{\mathcal{O}}_{f} \subseteq \mathcal{R}_{f}$ so, if $\alpha>0$ then $\alpha \in \Delta_{f}$.

On the other hand, if $\alpha<0$ then, by Lemma 3.3, there exists

$$
\omega=A d x+B d y=A_{0} d x+B_{0} d y+Q d f+P f d x \in f \cdot \Omega\left(\log C_{f}\right),
$$

with $\omega_{0}=A_{0} d x+B_{0} d y \in \mathcal{E}(f) \cap f \cdot \Omega\left(\log C_{f}\right), P, Q \in \mathbb{C}\{x, y\}$ and $M f=P_{f}(\omega)=P_{f}\left(\omega_{0}\right)+$ $P f_{y} f=\left(M_{0}+P f_{y}\right) f$. As $\alpha<0$ we have $\alpha+\mu_{f}-1+v_{0}<\nu_{f}\left(f_{y}\right) \leq \nu_{f}\left(P f_{y}\right)$ and, by Proposition 
3.7 $\nu_{f}(M)=\nu_{f}\left(M_{0}\right)=\nu_{f}\left(B_{0}\right)-v_{0}$, that is, $\nu_{f}\left(B_{0}\right)=\alpha+\mu_{f}-1+v_{0}<\nu_{f}\left(f_{y}\right)$. In this way, $\nu_{f}(B)=\nu_{f}\left(B_{0}+Q f_{y}\right)=\nu_{f}\left(B_{0}\right)$ and $\nu_{f}(\operatorname{res}(\omega))=\nu_{f}\left(\frac{B}{f_{y}}\right)=\nu_{f}\left(\frac{B_{0}}{f_{y}}\right)=\alpha \in \Delta_{f}$.

As a consequence of the considerations in the proof of the above theorem we have the following result:

Corollary 3.9. For every $\alpha \in \Delta_{f} \backslash \Gamma_{f}$ there exists $\omega \in \mathcal{E}(f) \cap f \cdot \Omega\left(\log C_{f}\right)$ such that $\nu_{f}(\operatorname{res}(\omega))=$ $\alpha=\nu_{f}\left(\frac{P_{f}(\omega)}{f}\right)-\left(\mu_{f}-1\right)$.

Proposition 3.7 relates $\nu_{f}(B)$ (equivalently $\nu_{f}(A)$ ) and $\nu_{f}(M)$ for $A \in \mathcal{P}_{n}$ and $B, M \in \mathcal{P}_{n-1}$ satisfying $A f_{y}+B f_{x}=M f$. As $f_{x}$ and $f_{y}$ are coprime, given $H \in \mathbb{K}[y]$ there exist $A, B \in \mathbb{K}[y]$ such that $A f_{y}+B f_{x}=H$. In [BMT], Briançon, Maisonobe and Torrelli consider such equation and, with the notations introduced before, obtain the following result:

Lemma 3.10 (Proposition 2.16, [BMT]). If $B=\sum_{i=1}^{n} B_{i} \Phi_{i}, H=\sum_{i=1}^{n} H_{i} \Phi_{i} \in \mathcal{P}_{n-1}^{K}$ and $A=$ $\sum_{i=1}^{n} A_{i} \Phi_{i} \in \mathcal{P}_{n}^{K}$ satisfy $A f_{y}+B f_{x}=H$ then $A=\sum_{i=1}^{n}\left(B_{i} \varphi_{i}^{\prime}+\frac{H_{i}}{\Phi_{i}\left(\varphi_{i}\right)}\right) \Phi_{i}$ and

$$
\begin{aligned}
\left(\sum_{\substack{j=1 \\
j \neq i}}^{n} \frac{\varphi_{i}^{\prime}-\varphi_{j}^{\prime}}{\varphi_{i}-\varphi_{j}}\right) \cdot B_{i}-\sum_{\substack{j=1 \\
j \neq i}}^{n}\left(\left(\frac{\varphi_{i}^{\prime}-\varphi_{j}^{\prime}}{\varphi_{i}-\varphi_{j}}\right) \cdot B_{j}\right) & =\left(\sum_{\substack{j=1 \\
j \neq i}}^{n} \frac{\Phi_{j}\left(\varphi_{j}\right)-\Phi_{i}\left(\varphi_{i}\right)}{\left(\varphi_{i}-\varphi_{j}\right) \Phi_{i}\left(\varphi_{i}\right) \Phi_{j}\left(\varphi_{j}\right)}\right) \cdot H_{i} \\
& -\sum_{\substack{j=1 \\
j \neq i}}^{n}\left(\left(\frac{\Phi_{j}\left(\varphi_{j}\right)-\Phi_{i}\left(\varphi_{i}\right)}{\left(\varphi_{i}-\varphi_{j}\right) \Phi_{i}\left(\varphi_{i}\right) \Phi_{j}\left(\varphi_{j}\right)}\right) \cdot H_{j}\right) .
\end{aligned}
$$

Using the computations presented in Proposition 3.7 we can relate $\nu_{f}(H)$ and $\nu_{f}(B)$.

Proposition 3.11. With the previous notation we get

$$
\nu_{f}(H) \leq \nu_{f}(B)+\mu_{f}-1+\beta_{g}
$$

where $\beta_{g}$ is the greatest characteristic exponent of $C_{f}$.

Proof: By (7), (8), (9) and (10) we have

$$
\begin{gathered}
\left.\left(\sum_{j=1}^{n-1} \frac{\varphi_{n}^{\prime}-\varphi_{j}^{\prime}}{\varphi_{n}-\varphi_{j}}\right) \cdot B_{n}-\sum_{j=1}^{n-1}\left(\left(\frac{\varphi_{n}^{\prime}-\varphi_{j}^{\prime}}{\varphi_{n}-\varphi_{j}}\right) \cdot B_{j}\right)=\frac{b S}{c n} x^{\frac{\nu_{f}(B)-\nu_{f}\left(f_{y}\right)-n}{n}}+\text { (h.o.t. }\right) \\
\frac{\Phi_{n}\left(\varphi_{n}\right)-\Phi_{j}\left(\varphi_{j}\right)}{\left(\varphi_{n}-\varphi_{j}\right) \Phi_{n}\left(\varphi_{n}\right) \Phi_{j}\left(\varphi_{j}\right)}=\left(\frac{1-\eta^{j \nu_{f}\left(f_{y}\right)}}{c u_{j} \eta^{j \nu_{f}\left(f_{y}\right)}}\right) x^{\frac{-\left(\nu_{f}\left(f_{y}\right)+m_{j}\right)}{n}}+\text { (h.o.t.)},
\end{gathered}
$$

where $\eta$ is a $n$-th primitive root of the unity, $m_{j} \in\left\{\beta_{1}, \ldots, \beta_{g}\right\}$ and $S \neq 0$ as showed in Proposition 3.7 
Denoting $H\left(x, \varphi_{j}\right)=h \eta^{j \nu_{f}(H)} x^{\frac{\nu_{f}(H)}{n}}+$ (h.o.t.) with $h \in \mathbb{C} \backslash\{0\}$ we obtain

$$
\begin{aligned}
\left(\sum_{j=1}^{n-1} \frac{\Phi_{j}\left(\varphi_{j}\right)-\Phi_{n}\left(\varphi_{n}\right)}{\left(\varphi_{n}-\varphi_{j}\right) \Phi_{n}\left(\varphi_{n}\right) \Phi_{j}\left(\varphi_{j}\right)}\right) \cdot H_{n}-\sum_{j=1}^{n-1}\left(\left(\frac{\Phi_{j}\left(\varphi_{j}\right)-\Phi_{n}\left(\varphi_{n}\right)}{\left(\varphi_{n}-\varphi_{j}\right) \Phi_{n}\left(\varphi_{n}\right) \Phi_{j}\left(\varphi_{j}\right)}\right) \cdot H_{j}\right)= \\
=\sum_{j=1}^{n}\left(\frac{1-\eta^{j \nu_{f}\left(f_{y}\right)}}{u_{j} \eta^{2 j \nu_{f}\left(f_{y}\right)}}\right)\left(\frac{h}{c^{2}} \eta^{j \nu_{f}(H)}\right) x^{\frac{\nu_{f}(H)-2 \nu_{f}\left(f_{y}\right)-m_{j}}{n}}+\text { (h.o.t.). }
\end{aligned}
$$

By the previous lemma, expressions (13) and (14) there exists $1 \leq j \leq n$ such that

$$
\frac{\nu_{f}(H)-2 \nu_{f}\left(f_{y}\right)-m_{j}}{n} \leq \frac{\nu_{f}(B)-\nu_{f}\left(f_{y}\right)-n}{n}
$$

that is, $\nu_{f}(H) \leq \nu_{f}(B)+\mu_{f}-1+m_{j} \leq \nu_{f}(B)+\mu_{f}-1+\beta_{g}$.

\section{Relating analytical invariants to $C_{k}$ and $C_{f}$}

Given a $k$-semiroot $f_{k}$ of $f$ with $0 \leq k<g$, by Lemma 3.3. we get $\Omega_{1}=\mathcal{E}\left(f_{k}\right) \oplus \mathcal{G}\left(f_{k}\right)$ with

$\mathcal{G}\left(f_{k}\right) \subseteq \mathcal{F}\left(f_{k}\right)$. In this way, for each $\varpi \in \Omega_{f_{k}}=\frac{\Omega^{1}}{\mathcal{F}\left(f_{k}\right)}$ there exist $\omega \in \mathcal{E}\left(f_{k}\right)$ such that $\bar{\omega}=\varpi$. In this section we will analyze $\nu_{f}(\omega)$ for $\omega \in \mathcal{E}\left(f_{k}\right)$ and we will describe elements in $\Lambda_{f} \backslash \Gamma_{f}$ from elements of $\Lambda_{k} \backslash \Gamma_{k}$.

Notice that for $k=0$, that is $f_{0}=y+a(x) \in \mathbb{C}\{x\}[y]$, we have $\mathcal{E}\left(f_{0}\right)=\mathbb{C}\{x\} d x+\mathbb{C}\{x\} d y$ and $\nu_{f}\left(\mathcal{E}\left(f_{0}\right)\right) \subseteq\left\{v_{0}+k v_{0}, v_{1}+k v_{0} ; k \in \mathbb{N}\right\} \subset \Gamma_{f}$.

In what follows we consider $1 \leq k<g$.

Given $\omega=A d x-B d y \in \mathcal{E}\left(f_{k}\right)$, that is, $A, B \in \mathbb{C}\{x\}[y]$ with $\operatorname{deg}_{y}(A)<\operatorname{deg}_{y}\left(f_{k}\right)-1=\frac{n}{e_{k}}-1$ and $\operatorname{deg}_{y}(B)<\frac{n}{e_{k}}$ we can write

$$
P_{f_{k}}(w)=A \cdot\left(f_{k}\right)_{y}+B \cdot\left(f_{k}\right)_{x}=H+M f_{k}
$$

with $H, M \in \mathbb{C}\{x\}[y], \operatorname{deg}_{y}(H)<\frac{n}{e_{k}}$ and $\operatorname{deg}_{y}(M)<\frac{n}{e_{k}}-1$.

We will consider the cases: $\omega \in \mathcal{E}\left(f_{k}\right) \cap f_{k} \cdot \Omega\left(\log C_{k}\right)$ or $\omega \in \mathcal{E}\left(f_{k}\right) \backslash f_{k} \cdot \Omega\left(\log C_{k}\right)$.

\subsection{Case: $\omega \in \mathcal{E}\left(f_{k}\right) \cap f_{k} \cdot \Omega\left(\log C_{k}\right)$}

In this case, $H=0$ in (15) and we obtain the following result:

Proposition 4.1. If $\omega=A d x-B d y \in \mathcal{E}\left(f_{k}\right) \cap f_{k} \cdot \Omega\left(\log C_{k}\right)$ with $P_{f_{k}}(w)=M f_{k}$ then

$$
\nu_{f}(\omega)=v_{k+1}-e_{k}\left(\mu_{k}-1-\nu_{k}(M)\right)=v_{k+1}-e_{k}\left(\mu_{k}-1+\frac{v_{0}}{e_{k}}-\nu_{f_{k}}(B)\right) .
$$

In particular, $\nu_{f}\left(\mathcal{E}\left(f_{k}\right) \cap f_{k} \cdot \Omega\left(\log C_{k}\right)\right) \subseteq\left\{v_{k+1}-e_{k} \delta>0 ; 0 \neq \delta \in \mathbb{Z} \backslash \Lambda_{k}\right\}$. 
ProOF: If $\left(t^{\frac{v_{0}}{e_{k}}}, \psi(t)\right)$ and $\left(t^{v_{0}}, \varphi(t)\right)$ denote parametrizations for $C_{k}$ and $C_{f}$ respectively, then by Proposition 2.6 and Proposition 3.7 there exists $0 \leq j \leq k-1$ such that

$$
\begin{array}{ll}
\left.M\left(t^{\frac{v_{0}}{e_{k}}}, \psi(t)\right)=\frac{e_{j} v_{j+1}}{n} b t^{\nu_{k}(M)}+\text { (h.o.t. }\right) ; & M\left(t^{v_{0}}, \varphi(t)\right)=\frac{e_{j} v_{j+1}}{n} b t^{\nu_{f}(M)}+\text { (h.o.t.); } \\
B\left(t^{\frac{v_{0}}{e_{k}}}, \psi(t)\right)=b t^{\nu_{k}(B)}+(\text { h.o.t. }) ; & B\left(t^{v_{0}}, \varphi(t)\right)=b t^{\nu_{f}(B)}+\text { (h.o.t.) }
\end{array}
$$

with $\nu_{f}(B)=e_{k} \nu_{k}(B)=e_{k}\left(\nu_{k}(M)+\frac{v_{0}}{e_{k}}\right)=\nu_{f}(M)+v_{0}$.

As $\operatorname{deg}_{y}\left(\left(f_{k}\right)_{y}\right)<\operatorname{deg}_{y}\left(f_{k}\right)$, Proposition 2.6 gives us $\nu_{f}\left(\left(f_{k}\right)_{y}\right)=e_{k} \nu_{k}\left(\left(f_{k}\right)_{y}\right)=e_{k}\left(\mu_{k}-1\right)+v_{0}$.

By (44) we get $\left(f_{k}\right)_{y} \omega=M f_{k} d x-B d f_{k}$. If $f_{k}\left(t^{v_{0}}, \varphi(t)\right)=a t^{v_{k+1}}+$ (h.o.t.) with $a \in \mathbb{C} \backslash\{0\}$ then $\left(M f_{k} d x-B d f_{k}\right)\left(t^{v_{0}}, \varphi(t)\right)=a b\left(e_{j} v_{j+1}-v_{k}\right) t^{\nu_{f}(B)}+$ (h.o.t.) and

$$
v_{f}(\omega)=v_{k+1}-e_{k}\left(\mu_{k}-1-\nu_{k}(M)\right)=v_{k+1}-e_{k}\left(\mu_{k}-1+\frac{v_{0}}{e_{k}}-\nu_{f_{k}}(B)\right) .
$$

In particular, by Theorem 3.8, $-\delta=\mu_{k}-1-\nu_{k}(M) \in \Delta_{k} \backslash\{0\}$, or equivalently, $\delta \notin \Lambda_{k}$. So, $\nu_{f}\left(\mathcal{E}\left(f_{k}\right) \cap f_{k} \cdot \Omega\left(\log C_{k}\right)\right) \subseteq\left\{v_{k+1}-e_{k} \delta>0 ; 0 \neq \delta \in \mathbb{Z} \backslash \Lambda_{k}\right\}$.

As a consequence we obtain the following corollary:

Corollary 4.2. Considering $\Gamma_{f}=\left\langle v_{0}, \ldots, v_{g}\right\rangle$ and maintaining the above notation we have

$$
\nu_{f}\left(\mathcal{E}\left(f_{k}\right) \cap f_{k} \cdot \Omega\left(\log C_{k}\right)\right) \cap\left(\Lambda_{f} \backslash \Gamma_{f}\right)=\left\{v_{k+1}-e_{k} \delta ; \delta \in \mathbb{N}^{*} \backslash \Lambda_{k} \text { or }-\delta \in \mathbb{N} \backslash \Gamma_{k}\right\} .
$$

Moreover, for every $\delta \in\left\{\alpha ; \alpha \in \mathbb{N}^{*} \backslash \Lambda_{k}\right\} \dot{\cup}\left\{-\alpha ; \alpha \in \mathbb{N} \backslash \Gamma_{k}\right\}$ we have

$$
\sum_{i=k+1}^{g} s_{i} v_{i}-e_{k} \delta \in \Lambda_{f} \backslash \Gamma_{f}
$$

where $0 \leq s_{j}<n_{j}, k<j \leq g$ with $s_{k+1} \neq 0$.

ProOF: Firstly, remark that for $-\delta \in \Gamma_{k}$ we have $v_{k+1}-e_{k} \delta \in \Gamma_{f}$, then by the above proposition we get $\nu_{f}\left(\mathcal{E}\left(f_{k}\right) \cap f_{k} \cdot \Omega\left(\log C_{k}\right)\right) \cap\left(\Lambda_{f} \backslash \Gamma_{f}\right) \subseteq\left\{v_{k}-e_{k} \delta ; \delta \in \mathbb{N}^{*} \backslash \Lambda_{k}\right.$ or $\left.-\delta \in \mathbb{N} \backslash \Gamma_{k}\right\}$.

On the other hand, if $\delta \in \mathbb{N}^{*} \backslash \Lambda_{k}$, respectively $-\delta \in \mathbb{N} \backslash \Gamma_{k}$ then, by Theorem 3.8 (applied for $f_{k}$ ), there exists $\omega_{0}=A_{0} d x+B_{0} d y \in \mathcal{E}\left(f_{k}\right) \cap f_{k} \cdot \Omega\left(\log C_{k}\right)$ (see (11), respectively (12)) such that $P_{f_{k}}\left(\omega_{0}\right)=M_{0} f_{k}$ with $\nu_{k}\left(M_{0}\right)=\mu_{k}-1-\delta$, consequently by the previous proposition we have $v_{k+1}-e_{k} \delta=v_{k+1}-e_{k}\left(\mu_{k}-1-\nu_{k}\left(M_{0}\right)\right)=\nu_{k}\left(\omega_{0}\right) \in \Lambda_{f} \cap \nu_{f}\left(\mathcal{E}\left(f_{k}\right) \cap f_{k} \cdot \Omega\left(\log C_{k}\right)\right)$.

Moreover, if $\left\{f_{0}, \ldots, f_{g}\right\}$ is a complete system of semiroots of $f$, then

$$
\gamma=\sum_{i=k+1}^{g} s_{i} v_{i}-e_{k} \delta=\nu_{f}\left(f_{k}^{s_{k+1}-1} \prod_{i=k+1}^{g-1} f_{i}^{s_{i+1}} \omega_{0}\right) \in \Lambda_{f}
$$

for $0 \leq s_{i}<n_{i}, k<i \leq g$ with $s_{k+1} \neq 0$. So, to obtain the proposition it is sufficient to show that $\gamma \notin \Gamma_{f}$ for every $\delta \in\left\{\alpha ; \alpha \in \mathbb{N}^{*} \backslash \Lambda_{k}\right\} \dot{\cup}\left\{-\alpha ; \alpha \in \mathbb{N} \backslash \Gamma_{k}\right\}$. Notice that, taking $s_{k+1}=1, s_{k+2}=\ldots=s_{g-1}=0$ we get (16). 
If $-\delta \in \mathbb{N} \backslash \Gamma_{k}$ then, by Remark 2.1, we can write $-\delta=\sum_{i=0}^{k} s_{i} \frac{v_{i}}{e_{k}}$ with $0 \leq s_{i}<n_{i} ; 1 \leq i \leq k$ and $s_{0}<0$, consequently $\gamma=\sum_{i=0}^{g} s_{i} v_{i} \notin \Gamma_{f}$.

If $\delta \in \mathbb{N}^{*} \backslash \Lambda_{k}$ and $v_{k+1}-e_{k} \delta \in \Gamma_{f}$, as $v_{k+1}-e_{k} \delta<v_{k+1}$ we must have that $e_{k}$ divides $v_{k+1}-e_{k} \delta$, that is, $e_{k} \mid v_{k+1}$ that is an absurd. So, by Remark 2.1, $v_{k+1}-e_{k} \delta=\sum_{i=0}^{k} s_{i} v_{i}$ with $0 \leq s_{i}<n_{i} ; 1 \leq i \leq k$ and $s_{0}<0$ and $\gamma=\sum_{i=0}^{k} s_{i} v_{i}+\left(s_{k+1}-1\right) v_{k+1}+\sum_{i=k+2}^{g} s_{i} v_{i} \notin \Gamma_{f}$.

\subsection{Case: $\omega \in \mathcal{E}\left(f_{k}\right) \backslash f_{k} \cdot \Omega\left(\log C_{k}\right)$}

With the same above notations we consider $\omega=A d x-B d y \in \mathcal{E}\left(f_{k}\right) \backslash f_{k} \cdot \Omega\left(\log C_{k}\right)$ and $\nu_{k}(\omega) \in \Lambda_{k}$. Remark that $P_{f_{k}}(\omega)=A \cdot\left(f_{k}\right)_{y}+B \cdot\left(f_{k}\right)_{x}=H+M f_{k}$ and

$$
\left(f_{k}\right)_{y} \omega=P_{f_{k}}(\omega) d x-B d f_{k}=H d x+M f_{k} d x-B d f_{k}
$$

with $B, H, M \in \mathbb{C}\{x\}[y], \operatorname{deg}_{y}(H)<\operatorname{deg}_{y}\left(f_{k}\right)=\frac{n}{e_{k}}$ and $\operatorname{deg}_{y}(B), \operatorname{deg}_{y}(M)<\frac{n}{e_{k}}-1$. As $\nu_{k}\left(\left(f_{k}\right)_{y}\right)=\mu_{k}-1+\frac{n}{e_{k}}$ we get

$$
\nu_{k}(\omega)=\nu_{k}(H)-\left(\mu_{k}-1\right)
$$

On the other hand, as $\left(f_{k}\right)_{y}$ and $\left(f_{k}\right)_{x}$ are coprime in $\mathbb{C}((x))[y]$ there exist $A^{\prime}, B^{\prime} \in \mathbb{C}((x))[y]$ with $\operatorname{deg}_{y}\left(A^{\prime}\right)<\frac{n}{e_{k}}$ and $\operatorname{deg}_{y}\left(B^{\prime}\right)<\frac{n}{e_{k}}-1$ such that

$$
A^{\prime} \cdot\left(f_{k}\right)_{y}+B^{\prime} \cdot\left(f_{k}\right)_{x}=M f_{k}
$$

In particular, there exists $Q \in \mathbb{C}\{x\}$ such that $A_{0}=Q A^{\prime}, B_{0}=Q B^{\prime} \in \mathbb{C}\{x\}[y]$ satisfy $A_{0} \cdot\left(f_{k}\right)_{y}+$ $B_{0} \cdot\left(f_{k}\right)_{x}=Q M f_{k}$ and, by Proposition 3.7, $\nu_{k}\left(B_{0}\right)=\nu_{k}(Q M)+\frac{n}{e_{k}}$ with $B_{0}\left(t^{\frac{v_{0}}{e_{k}}}, \varphi_{k}(t)\right)=$ $b t^{\nu_{k}\left(B_{0}\right)}+$ (h.o.t.), $Q M\left(t^{\frac{v_{0}}{e_{k}}}, \varphi_{k}(t)\right)=\frac{e_{j} v_{j} b}{v_{0}} t^{\nu_{k}(Q M)}+$ (h.o.t) where $e_{j}=\min \left\{e_{i} ; e_{i} \nmid \nu_{k}\left(B_{0}\right)-\right.$ $\left.\nu_{k}\left(\left(f_{k}\right)_{y}\right)\right\}<e_{k}$ and $\left(t^{\frac{v_{0}}{e_{k}}}, \varphi_{k}(t)\right)$ is a parametrization of $f_{k}$. Consequently, by Proposition 2.6, we have

$$
\nu_{f}\left(B^{\prime}\right)+v_{k+1}=\nu_{f}(M)+v_{0}+v_{k+1}=\nu_{f}\left(M f_{k} d x-B^{\prime} d f_{k}\right) .
$$

In this way, $\left(A-A^{\prime}\right) \cdot\left(f_{k}\right)_{y}+\left(B-B^{\prime}\right) \cdot\left(f_{k}\right)_{x}=H$ and, by Proposition $3.11 \nu_{k}(H) \leq \nu_{k}(B-$ $\left.B^{\prime}\right)+\mu_{k}-1+\frac{\beta_{k}}{e_{k}}$, or equivalently, by Proposition 2.6 $\nu_{f}(H)+v_{0} \leq \nu_{f}\left(B-B^{\prime}\right)+e_{k}\left(\mu_{k}-1\right)+\beta_{k}<$ $\nu_{f}\left(B-B^{\prime}\right)+e_{k}\left(\mu_{k}-1\right)+\beta_{k+1}$. Using (2) and (1) for $f_{k}$ we obtain

$$
\nu_{f}(H)+v_{0}<\nu_{f}\left(B-B^{\prime}\right)+v_{k+1} .
$$

Notice that $\nu_{f}(H)+v_{0}=e_{k}\left(\nu_{k}(H)+\frac{v_{0}}{e_{k}}\right)$ and $e_{k} \nmid\left(\nu_{f}(B)+v_{k+1}\right)$ so, $\nu_{f}(H)+v_{0} \neq \nu_{f}(B)+v_{k+1}$.

Lemma 4.3. Considering (17) and the above notations we have that

$$
\nu_{f}(\omega)=\left\{\begin{array}{lll}
e_{k} \nu_{k}(\omega) & \text { if } \quad \nu_{f}(H)+v_{0}<\nu_{f}(B)+v_{k+1} \\
e_{k} \nu_{k}(B)+\beta_{k+1} & \text { if } & \nu_{f}(H)+v_{0}>\nu_{f}(B)+v_{k+1} .
\end{array}\right.
$$


Proof: By (17) we have that

$\nu_{f}(\omega)=\nu_{f}\left(H d x+M f_{k} d x-B d f_{k}\right)-\nu_{f}\left(\left(f_{k}\right)_{y}\right)=\nu_{f}\left(H d x+M f_{k} d x-B d f_{k}\right)-e_{k}\left(\mu_{k}-1+\frac{v_{0}}{e_{k}}\right)$.

Suppose $\nu_{f}(H)+v_{0}<\nu_{f}(B)+v_{k+1}$. If $\nu_{f}\left(B-B^{\prime}\right)=\min \left\{\nu_{f}(B), \nu_{f}\left(B^{\prime}\right)\right\}$, then $\nu_{f}\left(B-B^{\prime}\right)+$ $v_{k+1} \leq \nu_{f}\left(B^{\prime}\right)+v_{k+1}=\nu_{f}(M)+v_{0}+v_{k+1}$. If $\nu_{f}\left(B-B^{\prime}\right)>\min \left\{\nu_{f}(B), \nu_{f}\left(B^{\prime}\right)\right\}$, then by (20) we get $\nu_{f}\left(M f_{k} d x-B d f_{k}\right)=\nu_{f}(B)+v_{k+1}$ and by (18) $\nu_{f}\left(\left(f_{k}\right)_{y} \omega\right)=\nu_{f}(H d x)=e_{k} \nu_{k}\left(\left(f_{k}\right)_{y} \omega\right)$.

Now suppose that $\nu_{f}(H)+v_{0}>\nu_{f}(B)+v_{k+1}$. As $\nu_{f}(H)+v_{0}<\nu_{f}\left(B-B^{\prime}\right)+v_{k+1}$ we must have $\nu_{f}\left(B-B^{\prime}\right)>\min \left\{\nu_{f}(B), \nu_{f}\left(B^{\prime}\right)\right\}$, then by (20) we get $\nu_{f}\left(M f_{k} d x-B d f_{k}\right)=\nu_{f}(B)+v_{k+1}$ and $\nu_{f}(\omega)=\nu_{f}\left(B d f_{k}\right)-e_{k}\left(\mu_{k}-1+\frac{v_{0}}{e_{k}}\right)=v_{k+1}-e_{k}\left(\mu_{k}-1+\frac{v_{0}}{e_{k}}-\nu_{k}(B)\right)=\beta_{k+1}+e_{k} \nu_{k}(B)$ where the last equality follows because $e_{k}\left(\mu_{k}-1\right)+v_{0}=n_{k} v_{k}-\beta_{k}=v_{k+1}-\beta_{k+1}$.

Remark 4.4. Notice that by the previous result if $\nu_{f}(H)+v_{0}<\nu_{f}(B)+v_{k+1}$ then $\nu_{f}(\omega)=$ $e_{k} \nu_{k}(\omega)$ and consequently, $\nu_{f}(\omega) \in \Lambda_{f} \backslash \Gamma_{f}$ if, and only if, $\nu_{k}(\omega) \in \Lambda_{k} \backslash \Gamma_{k}$. In addition, as $\nu_{k}(\omega) \leq \mu_{k}-1$ we have that $\nu_{f}(\omega) \leq e_{k}\left(\mu_{k}-1\right)<v_{k+1}$.

If $\nu_{f}(H)+v_{0}>\nu_{f}(B)+v_{k+1}$, then $\nu_{k}(B)-\frac{v_{0}}{e_{k}}=\nu_{k}\left(B^{\prime}\right)-\frac{v_{0}}{e_{k}}=\nu_{k}(M) \in \Gamma_{k}$ and $\alpha=$ $\mu_{k}-1+\frac{v_{0}}{e_{k}}-\nu_{k}(B) \notin \Gamma_{k}$.

If $\alpha \in \Lambda_{k} \backslash \Gamma_{k}$, then similarly to Corollary 4.2 we get $v_{k+1}-e_{k} \alpha=\beta_{k+1}+e_{k} \nu_{k}(B) \in \Lambda_{f} \backslash \Gamma_{f}$.

On the other hand if $\alpha \notin \Lambda_{k} \backslash \Gamma_{k}$, by Theorem 3.8 we have $\nu_{k}(M) \in \nu_{k}\left(J\left(f_{k}\right): f_{k}\right)$. So, by Proposition 4.1. there exists $\omega^{\prime} \in \mathcal{E}\left(f_{k}\right) \cap f_{k} \cdot \Omega\left(\log C_{k}\right)$ such that $\nu_{f}\left(\omega^{\prime}\right)=v_{k+1}-e_{k} \alpha=\nu_{f}(\omega)$.

Remark 4.5. As $e_{k}\left(\mu_{k}-1\right)+v_{0}=v_{k+1}-\beta_{k+1}$ and, by (18), we have $\nu_{k}(H)=\nu_{k}(\omega)+\mu_{k}-1$ it follows that

$$
\nu_{f}(H)+v_{0}<\nu_{f}(B)+v_{k+1} \quad \Leftrightarrow \quad e_{k}\left(\nu_{k}(\omega)-\nu_{k}(B)\right)<\beta_{k+1} .
$$

Our aim is to define an injective function $\rho_{k}: \Lambda_{k} \backslash \Gamma_{k} \rightarrow \Lambda_{f} \backslash \Gamma_{f}$.

The previous lemma give us a way to relate such sets, but it depends on the expression of 1-form. For instance, if $\delta_{k}=\nu_{k}(A d x-B d y) \in \Lambda_{k} \backslash \Gamma_{k}$ is such $e_{k}\left(\delta_{k}-\nu_{k}(B)\right)<\beta_{k+1}$ then we can have $\omega_{i}=A_{i} d x-B_{i} d y \in \mathcal{E}\left(f_{k}\right) \backslash f_{k} \cdot \Omega\left(\log C_{k}\right)$ with $i=1,2, \nu_{k}\left(\omega_{1}\right)=\delta_{k}=\nu_{k}\left(\omega_{2}\right)$ and $\nu_{k}\left(B_{1}\right) \neq \nu_{k}\left(B_{2}\right)$.

In order to deal with this situation we consider the following function considered by Delorme in $[\mathrm{De}$ :

$$
\begin{aligned}
\Theta_{k}: \Lambda_{k} & \rightarrow \Gamma_{k} \cup\{\infty\} \\
\delta_{k} & \mapsto \max \left\{\nu_{k}(B) ; \delta_{k}=\nu_{k}(A d x-B d y)\right\} .
\end{aligned}
$$

Remark 4.6. The function $\Theta_{k}$ is not injective because $\Theta_{k}\left(\delta_{k}\right)=\infty$ for every $\delta_{k}=\nu_{k}(A d x) \in$ $\Gamma_{k}+\frac{v_{0}}{e_{k}} \subset \Gamma_{k}$.

In addition if $\delta_{k}=\sum_{i=1}^{k} s_{i} \frac{v_{i}}{e_{k}}$, then $\delta_{k}=\nu_{k}\left(d\left(\prod_{i=1}^{k} f_{i-1}^{s_{i}}\right)\right)$ where $\left\{f_{0}, \ldots, f_{k}\right\}$ is a complete system of semiroots of $f_{k}$ and $0 \leq s_{i}<n_{i}$ for $i=1, \ldots, k$. As $\nu_{k}\left(\prod_{i=1, i \neq j}^{k} f_{i-1}^{s_{i}} f_{j-1}^{s_{j}-1}\left(f_{j-1}\right)_{y}\right)=$ $\delta_{k}-\frac{\beta_{j}}{e_{k}}$ for $s_{j} \neq 0$, we have that $\Theta_{k}\left(\delta_{k}\right) \geq \delta_{k}-\frac{\beta_{r}}{e_{k}}$ where $r=\max \left\{j ; s_{j} \neq 0\right\}$. 
However, $\Theta_{k}: \Lambda_{k} \backslash \Gamma_{k} \rightarrow \Gamma_{k}$ is injective. In fact, given $\delta_{1}, \delta_{2} \in \Lambda_{k} \backslash \Gamma_{k}$ with $\delta_{1}<\delta_{2}$ if $\Theta_{k}\left(\delta_{1}\right)=\Theta_{k}\left(\delta_{2}\right)$, then there exist $\omega_{i}=A_{i} d x-B_{i} d y$ with $\nu_{k}\left(\omega_{i}\right)=\delta_{i}$ and $\nu_{k}\left(B_{i}\right)=\Theta\left(\delta_{i}\right)$ for $i=1,2$. In this way, there exists $c \in \mathbb{C}$ such that $\nu_{k}\left(B_{1}-c B_{2}\right)>\nu_{k}\left(B_{2}\right)=\nu_{k}\left(B_{1}\right)=\Theta_{k}\left(\delta_{1}\right)$ and considering $\omega=\omega_{1}-c \omega_{2}=\left(A_{1}-c A_{2}\right) d x-\left(B_{1}-c B_{2}\right) d y$ we get $\nu_{k}(\omega)=\nu_{k}\left(\omega_{1}\right)=\delta_{1}$ and $\Theta_{k}\left(\delta_{1}\right) \geq \nu_{k}\left(B_{1}-c B_{2}\right)>\Theta_{k}\left(\delta_{1}\right)$ that is an absurd.

We define

$$
\begin{aligned}
\rho_{k}: \Lambda_{k} \backslash \Gamma_{k} & \rightarrow \Lambda_{f} \backslash \Gamma_{f} \\
\delta_{k} & \mapsto\left\{\begin{array}{lll}
e_{k} \delta_{k} & \text { if } & e_{k}\left(\delta_{k}-\Theta_{k}\left(\delta_{k}\right)\right)<\beta_{k+1} \\
\beta_{k+1}+e_{k} \Theta_{k}\left(\delta_{k}\right) & \text { if } & e_{k}\left(\delta_{k}-\Theta_{k}\left(\delta_{k}\right)\right)>\beta_{k+1} .
\end{array}\right.
\end{aligned}
$$

As $\Theta_{k}: \Lambda_{k} \backslash \Gamma_{k} \rightarrow \Gamma_{k}$ is well defined and injective, by Remark 4.4 $\rho_{k}$ is well defined and injective also.

In what follows we denote

$L_{k}^{1}=\left\{\delta_{k} \in \Lambda_{k} \backslash \Gamma_{k} ; e_{k}\left(\delta_{k}-\Theta_{k}\left(\delta_{k}\right)\right)<\beta_{k+1}\right\}$ and $L_{k}^{2}=\left\{\delta_{k} \in \Lambda_{k} \backslash \Gamma_{k} ; e_{k}\left(\delta_{k}-\Theta_{k}\left(\delta_{k}\right)\right)>\beta_{k+1}\right\}$.

The following proposition summarizes the discussion of this subsection.

Proposition 4.7. With the notations introduced in this subsection we have that $\rho_{k}\left(L_{k}^{1}\right)=e_{k} L_{k}^{1} \subset$ $\Lambda_{f} \backslash \Gamma_{f}$ and

$$
\rho_{k}\left(L_{k}^{2}\right)=\left\{\beta_{k+1}+e_{k} \Theta_{k}\left(\delta_{k}\right)=v_{k+1}-e_{k}\left(\mu_{k}-1+\frac{v_{0}}{e_{k}}-\Theta_{k}\left(\delta_{k}\right)\right) ; \delta_{k} \in L_{k}^{2}\right\} \subset \Lambda_{f} \backslash \Gamma_{f} .
$$

In addition, $\sum_{i=k+1}^{g} s_{i} v_{i}+\rho_{k}\left(L_{k}^{2}\right) \subset \Lambda_{f} \backslash \Gamma_{f}$ for $0 \leq s_{i}<n_{i}$ for $i=k+2, \ldots, g$ and $0 \leq s_{k+1} \leq$ $n_{k+1}-2$.

Proof: The result follows directly form the above analysis and, similarly the computations done in Corollary 4.2, we get $\sum_{i=k+1}^{g} s_{i} v_{i}+\rho_{k}\left(L_{k}^{2}\right) \subset \Lambda_{f} \backslash \Gamma_{f}$ for $0 \leq s_{i}<n_{i}$ for $i=k+2, \ldots, g$ and $0 \leq s_{k+1} \leq n_{k+1}-2$.

\subsection{Main results}

The results presented in this section allow us to determine elements in $\Lambda_{f} \backslash \Gamma_{f}$ by $\Lambda_{k} \backslash \Gamma_{k}$ and consequently to relate the Tjurina number $\tau_{f}$ of $C_{f}$ with the Tjurina number $\tau_{k}$ of $C_{k}$.

Theorem 4.8. For any branch $C_{f}$ such that $f_{k}$ is a $k$-semiroot of $f$ with $0 \leq k<g$ we have

$$
e_{k} L_{k}^{1} \dot{\cup}\left\{\sum_{i=k+1}^{g} s_{i} v_{i}+\rho_{k}\left(L_{k}^{2}\right)\right\} \dot{\cup}\left\{\sum_{i=k+1}^{g} s_{i} v_{i}+v_{k+1}-e_{k} \delta\right\} \subseteq \Lambda_{f} \backslash \Gamma_{f}
$$

with

$0 \leq s_{i}<n_{i} ; i=k+2, \ldots, g, 0 \leq s_{k+1} \leq n_{k+1}-2$ and $\delta \in\left\{\alpha, \alpha \in \mathbb{N}^{*} \backslash \Lambda_{k}\right\} \dot{\cup}\left\{-\alpha, \alpha \in \mathbb{N} \backslash \Gamma_{k}\right\}$ 
Proof: By Corollary 4.2 and Proposition 4.7 we have

$$
\left\{\sum_{i=k+1}^{g} s_{i} v_{i}+v_{k+1}-e_{k} \delta\right\} \subseteq \Lambda_{f} \backslash \Gamma_{f} \quad \text { and } \quad e_{k} L_{k}^{1} \dot{\cup}\left\{\sum_{i=k+1}^{g} s_{i} v_{i}+\rho_{k}\left(L_{k}^{2}\right)\right\} \subseteq \Lambda_{f} \backslash \Gamma_{f} .
$$

It is immediate that $\left\{\sum_{i=k+1}^{g} s_{i} v_{i}+v_{k+1}-e_{k} \delta\right\} \cap e_{k} L_{k}^{1}=\emptyset$.

If $\left\{\sum_{i=k+1}^{g} s_{i} v_{i}+\rho_{k}\left(L_{k}^{2}\right)\right\} \cap\left\{\sum_{i=k+1}^{g} s_{i} v_{i}+v_{k+1}-e_{k} \delta\right\} \neq \emptyset$ then we must have

$$
\sum_{i=k+1}^{g} s_{i} v_{i}+v_{k+1}-e_{k} \gamma=\sum_{i=k+1}^{g} r_{i} v_{i}+v_{k+1}-e_{k} \delta
$$

with $v_{k+1}-e_{k} \gamma \in \rho_{k}\left(L_{k}^{2}\right), 0 \leq r_{i}, s_{i}<n_{i} ; i=k+2, \ldots, g, 0 \leq r_{k+1}, s_{k+1} \leq n_{k+1}-2$. Вy Remark 2.1, we get $\gamma=\delta$.

On the other hand, by Proposition 4.1 and Proposition 4.7 there exist $\omega_{1}=A_{1} d x-B_{1} d y \in$ $\mathcal{E}\left(f_{k}\right) \cap f_{k} \cdot \Omega\left(\log C_{k}\right)$ and $\omega_{2}=A_{2} d x-B_{2} d y \in \mathcal{E}\left(f_{k}\right) \backslash f_{k} \cdot \Omega\left(\log C_{k}\right)$ such that $\mu_{k}-1+\frac{v_{0}}{e_{k}}-\nu_{k}\left(B_{1}\right)=$ $\delta=\gamma=\mu_{k}-1+\frac{v_{0}}{e_{k}}-\nu_{k}\left(B_{2}\right)$. So, $\nu_{k}\left(B_{1}\right)=\nu_{k}\left(B_{2}\right)=\Theta_{k}\left(\nu_{k}\left(\omega_{2}\right)\right)$.

Taking $c \in \mathbb{C}$ such that $\nu_{k}\left(B_{1}-c B_{2}\right)>\nu_{k}\left(B_{1}\right)=\nu_{k}\left(B_{2}\right)$ we obtain $\omega=\omega_{1}-c \omega_{2} \in \mathcal{E}\left(f_{k}\right) \backslash f_{k}$. $\Omega\left(\log f_{k}\right)$ with $\nu_{k}(\omega)=\nu_{k}\left(\omega_{2}\right)$ and $\nu_{k}\left(B_{2}\right)=\Theta_{k}\left(\nu_{k}\left(\omega_{2}\right)\right)=\Theta_{k}\left(\nu_{k}(\omega)\right) \geq \nu_{k}\left(B_{1}-c B_{2}\right)>\nu_{k}\left(B_{2}\right)$, that is an absurd.

Hence, $\left\{\sum_{i=k+1}^{g} s_{i} v_{i}+\rho_{k}\left(L_{k}^{2}\right)\right\} \cap\left\{\sum_{i=k+1}^{g} s_{i} v_{i}+v_{k+1}-e_{k} \delta\right\}=\emptyset$ and we conclude the proof.

For any branch $C_{f}$ we have that $\tau_{f}=\mu_{f}-\sharp\left(\Lambda_{f} \backslash \Gamma_{f}\right)$ and consequently we obtain the following corollary.

Corollary 4.9. For any branch $C_{f}$ such that $f_{k}$ is a $k$-semiroot of $f$ with $0 \leq k<g$ we have $\sharp\left(\Lambda_{f} \backslash \Gamma_{k}\right) \geq \mu_{k}+e_{k+1}\left(n_{k+1}-2\right) \tau_{k}$. In particular, $\tau_{f} \leq \mu_{f}-\mu_{k}-e_{k+1}\left(n_{k+1}-2\right) \tau_{k}$.

ProOF: Notice that $\Lambda_{k} \backslash \Gamma_{k}=L_{k}^{1} \dot{\cup} L_{k}^{2}, \sharp\left(\rho_{k}\left(L_{k}^{1}\right)\right)=\sharp\left(L_{k}^{1}\right)$,

$\sharp\left\{\sum_{i=k+1}^{g} s_{i} v_{i}+\rho_{k}\left(L_{k}^{2}\right)\right\}=e_{k+1}\left(n_{k+1}-1\right) \sharp\left(L_{k}^{2}\right)$ and $\sharp\left\{\sum_{i=k+1}^{g} s_{i} v_{i}+v_{k+1}-e_{k} \delta\right\}=e_{k+1}\left(n_{k+1}-1\right) \cdot \sharp(T)$

with

$0 \leq s_{i}<n_{i} ; i=k+2, \ldots, g, 0 \leq s_{k+1} \leq n_{k+1}-2$ and $\delta \in T:=\left\{\alpha, \alpha \in \mathbb{N}^{*} \backslash \Lambda_{k}\right\} \dot{\cup}\left\{-\alpha, \alpha \in \mathbb{N} \backslash \Gamma_{k}\right\}$

As $\sharp\left(\mathbb{N} \backslash \Gamma_{k}\right)=\frac{\mu}{2}$ (see (22) ) and $\mathbb{N}^{*} \backslash \Lambda_{k}=\left(\mathbb{N} \backslash \Gamma_{k}\right) \backslash\left(\Lambda_{k} \backslash \Gamma_{k}\right)$ we get $\sharp(T)=\mu_{k}-\sharp\left(\Lambda_{k} \backslash \Gamma_{k}\right)=\tau_{k}$.

By the previous theorem we obtain

$$
\begin{aligned}
\sharp\left(\Lambda_{f} \backslash \Gamma_{f}\right) & \left.\geq \sharp\left(L_{k}^{1}\right)\right)+e_{k+1}\left(n_{k+1}-1\right)\left(\sharp\left(L_{k}^{2}\right)+\tau_{k}\right) \\
& =\sharp\left(L_{k}^{1}\right)+\sharp\left(L_{k}^{2}\right)+\tau_{k}+e_{k+1}\left(n_{k+1}-2\right)\left(\sharp\left(L_{k}^{2}\right)+\tau_{k}\right) \\
& =\sharp\left(\Lambda_{k} \backslash \Gamma_{k}\right)+\tau_{k}+e_{k+1}\left(n_{k+1}-2\right)\left(\sharp\left(L_{k}^{2}\right)+\tau_{k}\right) \\
& =\mu_{k}+e_{k+1}\left(n_{k+1}-2\right)\left(\sharp\left(L_{k}^{2}\right)+\tau_{k}\right) \geq \mu_{k}+e_{k+1}\left(n_{k+1}-2\right) \tau_{k} .
\end{aligned}
$$


In particular, as $\tau_{f}=\mu_{f}-\sharp\left(\Lambda_{f} \backslash \Gamma_{f}\right)$ we get $\tau_{f} \leq \mu_{f}-\mu_{k}-e_{k+1}\left(n_{k+1}-2\right) \tau_{k}$.

As $\frac{3 \mu_{f}}{4} \leq \tau_{f}$ for any branch $C_{f}$ (see [AABM], [GH] and [Wan] considering the semiroot $f_{g-1}$, the previous results give us an upper bound for $\tau_{f}$ in terms of topological data.

Corollary 4.10. For any branch $C_{f}$ with semigroup $\Gamma_{f}=\left\langle v_{0}, \ldots, v_{g}\right\rangle$ we have $\tau_{f} \leq \mu_{f}-$ $\frac{\left(3 n_{g}-2\right)}{4} \mu_{g-1}$.

Proof: As $e_{g}=1$, by Corollary 4.9 and using that $\frac{3 \mu_{g-1}}{4} \leq \tau_{g-1}$ we obtain the result.

Remark 4.11. In $\mid \underline{G H}]$, the authors proof that $\tau_{f} \geq \frac{3}{4} \mu_{f}+\left(\frac{-1+\sqrt{1+4 \mu_{f}}}{8}\right)$ for any irreducible plane curve $C_{f}$. So, we can use this inequality for $f_{g-1}$ to obtain a finer topological upper bound for $\tau_{f}$ in the above corollary.

Example 4.12. In [HH1] the authors consider branches $C_{f}$ with semigroup $\Gamma=\langle 6,9,19\rangle$ and they present all possibilities for $\Lambda_{f} \backslash \Gamma_{f}$ and consequently all possible values for $\tau_{f}$.

Any branch admitting this semigroup $\Gamma$ can be done by a parametrization $\left(t^{6}, t^{9}+a t^{9}+(\right.$ h.o.t. $\left.)\right)$ with $a \neq 0$ and all of them share the semiroot $f_{1}=y^{2}-x^{3}$ with $\Gamma_{1}=\langle 2,3\rangle, \Lambda_{1} \backslash \Gamma_{1}=\emptyset$ and $\tau_{1}=\mu_{1}=2$.

Considering $k=1$ in Theorem 4.8, we obtain that $\{16,22,35,41\} \subseteq \Lambda_{f} \backslash \Gamma_{f}$ and by Corollary 4.9 (or Corollary 4.10) $\tau_{f} \leq 38$ for any branch $C_{f}$. In fact, in [HH1] we found the following possibilites:

\begin{tabular}{|c|c|}
\hline$\Lambda \backslash \Gamma$ & $\tau$ \\
\hline$\{16,22,26,29,32,35,41\}$ & 35 \\
\hline$\{16,22,26,32,35,41\}$ & 36 \\
\hline$\{16,22,29,32,35,41\}$ & 36 \\
\hline$\{16,22,29,35,41\}$ & 37 \\
\hline
\end{tabular}

Notice that in above example we get $n_{g}=n_{2}=3$. In the next section we will show that for semigroup $\Gamma=\left\langle v_{0}, \ldots, v_{g}\right\rangle$ with $n_{g}=2$ we obtain the equality in Corollary 4.10 .

\section{Branches with semigroup $\Gamma=\left\langle v_{0}, \ldots, v_{g}\right\rangle$ and $n_{g}=2$}

In [LP], Luengo and Pfister proof that in the topological class determined by semigroup $\Gamma=$ $\left\langle v_{0}, v_{1}, v_{2}\right\rangle$ with $n_{2}=G C D\left(v_{0}, v_{1}\right)=2$ the Tjurina number $\tau_{f}$ is the same for any branch $C_{f}$ belonging to it and $\tau_{f}=\mu_{f}-\left(\frac{v_{0}}{2}-1\right)\left(\frac{v_{1}}{2}-1\right)=\mu_{f}-\mu_{1}$ where, as before, $\mu_{1}$ denote the Milnor number of the 1-semiroot $f_{1}$. Inspired by the result of Luengo and Pfister, Watari (see Wat]) ask if the Tjurina number can be expressed using topological data for branches with semigroup $\Gamma=\left\langle v_{0}, v_{1}, \ldots, v_{g}\right\rangle$ with $n_{i}=2$ for $i=1, \ldots, g$. 
In this section we will show that the inequality in Corollary 4.10 is sharp for any branch $C_{f}$ in the equisingularity class determined by $\Gamma=\left\langle v_{0}, v_{1}, \ldots, v_{g}\right\rangle$ with $n_{g}=2$, that is, $\tau_{f}=\mu_{f}-\mu_{g-1}$. In particular, we obtain a generalization of the result of Luengo-Pfister and an answer for a Watari's question.

Notice that for $g=1$ if $n_{1}=2$ then we get $\Gamma=\left\langle 2, v_{1}\right\rangle$ and $\tau_{f}=\mu_{f}=v_{1}-1$ for any curve $C_{f}$ with semigroup $\Gamma$ (see [Z2] for instance). So, in what follows we will suppose that $g>1$.

Firstly we present a result for any branch with semigroup $\Gamma=\left\langle v_{0}, \ldots, v_{g}\right\rangle$ without restriction on $n_{i}$.

Proposition 5.1. With the same notation as introduced in previous section and $1 \leq k<g$ we have that

$$
\left\{\lambda \in \Lambda_{f} \backslash \Gamma_{f} ; \lambda<v_{k+1}\right\}=\rho_{k}\left(\Lambda_{k} \backslash \Gamma_{k}\right) \dot{\cup}\left\{v_{k+1}-e_{k} \delta_{k} ; \delta_{k} \in \mathbb{N}^{*} \backslash \Lambda_{k}\right\} .
$$

Proof: By Corollary 4.2, using the map $\rho_{k}$ defined in (21) and Theorem 4.8 we get

$$
\rho_{k}\left(\Lambda_{k} \backslash \Gamma_{k}\right) \cup\left\{v_{k+1}-e_{k} \delta_{k} ; \delta_{k} \in \mathbb{N}^{*} \backslash \Lambda_{k}\right\} \subseteq\left\{\lambda \in \Lambda_{f} \backslash \Gamma_{f} ; \lambda<v_{k+1}\right\}
$$

To guarantee the other inclusion we consider $\lambda=\nu_{f}(\omega) \in \Lambda_{f} \backslash \Gamma_{f}$ with $\lambda<v_{k+1}$ with $\omega \in \Omega^{1}$. In particular, we can assume that $\omega \in \mathcal{E}\left(f_{k}\right) \backslash \mathcal{F}\left(f_{k}\right)$.

If $\omega \in \mathcal{E}\left(f_{k}\right) \cap f_{k} \cdot \Omega\left(\log C_{k}\right)$, by Corollary 4.2, we have that $\nu_{f}(\omega)=v_{k+1}-e_{k} \delta_{k}$ with $\delta_{k} \in \mathbb{N}^{*} \backslash \Lambda_{k}$.

Suppose that $\omega=A d x+B d y \in \mathcal{E}\left(f_{k}\right) \backslash f_{k} \cdot \Omega\left(\log C_{k}\right)$.

If $e_{k}\left(\nu_{k}(\omega)-\nu_{k}(B)\right)<\beta_{k+1}$ then, by Remark 4.4 and Remark 4.5, we obtain $\lambda=e_{k} \nu_{k}(\omega) \in$ $\rho_{k}\left(L_{k}^{1}\right) \subseteq \rho_{k}\left(\Lambda_{k} \backslash \Gamma_{k}\right)$.

On the other hand if $e_{k}\left(\nu_{k}(\omega)-\nu_{k}(B)\right)>\beta_{k+1}$, by Lemma 4.3 and Remark 4.5 we have $\lambda=v_{k+1}-e_{k} \alpha$ with $0<\alpha=\mu_{k}-1+\frac{v_{0}}{e_{k}}-\nu_{k}(B) \notin \Gamma_{k}$.

If $\alpha \notin \Lambda_{k}$ then $\lambda=v_{k+1}-e_{k} \alpha \in\left\{v_{k+1}-e_{k} \delta_{k} ; \delta_{k} \in \mathbb{N}^{*} \backslash \Lambda_{k}\right\}$.

The remain case to consider is $\omega=A d x+B d y \in \mathcal{E}\left(f_{k}\right) \backslash f_{k} \cdot \Omega\left(\log C_{k}\right)$ with $e_{k}\left(\nu_{k}(\omega)-\nu_{k}(B)\right)>$ $\beta_{k+1}, \nu_{k}(\omega)=\lambda=v_{k+1}-e_{k} \alpha$ and $0<\alpha=\mu_{k}-1+\frac{v_{0}}{e_{k}}-\nu_{k}(B) \in \Lambda_{k} \backslash \Gamma_{k}$. In particular, $\nu_{k}(B)-\frac{v_{0}}{e_{k}} \in \Gamma_{k}$, that is, $\nu_{k}(B) \in \Gamma_{k}+\frac{v_{0}}{e_{k}}$.

We will show that $\lambda \in \rho_{k}\left(L_{k}^{2}\right) \subset \rho_{k}\left(\Lambda_{k} \backslash \Gamma_{k}\right)$.

As $\nu_{k}(B) \in \Gamma_{k}+\frac{v_{0}}{e_{k}}$, if $\nu_{k}(B)=\Theta_{k}\left(\nu_{k}(\omega)\right)$ then $\nu_{k}(\omega) \in \Lambda_{k} \backslash \Gamma_{k}$. In fact, if $\nu_{k}(\omega) \in \Gamma_{k}$ then, by Remark 4.6, we get $\nu_{k}(B)=\Theta_{k}\left(\nu_{k}(\omega)\right)=\infty$ or $\nu_{k}(B)=\Theta_{k}\left(\nu_{k}(\omega)\right) \geq \nu_{k}(\omega)-\frac{\beta_{k}}{e_{k}}$, that is, $e_{k}\left(\nu_{k}(\omega)-\nu_{k}(B)\right) \leq \beta_{k}<\beta_{k+1}$ in both situations we have a contradiction.

In this way, if $\nu_{k}(B)=\Theta_{k}\left(\nu_{k}(\omega)\right)$ then $\nu_{k}(\omega) \in L_{k}^{2} \subset \Lambda_{k} \backslash \Gamma_{k}$ and consequently $\gamma=v_{k+1}-$ $e_{k} \alpha \in \rho_{k}\left(L_{k}^{2}\right) \subset \rho_{k}\left(\Lambda_{k} \backslash \Gamma_{k}\right)$.

To conclude the proof we consider $\nu_{k}(B)<\Theta_{k}\left(\nu_{k}(\omega)\right)$ and $\omega_{0}=A_{0} d x-B_{0} d y \in \mathcal{E}\left(f_{k}\right) \backslash f_{k}$. $\Omega\left(\log C_{f}\right)$ with $\nu_{k}\left(\omega_{0}\right)=\nu_{k}(\omega)=\delta_{0} \in \Lambda_{k} \backslash \Gamma_{k}, \nu_{k}\left(B_{0}\right)=\Theta_{k}\left(\delta_{0}\right)$ and $\delta_{1}=\nu_{k}\left(\omega-\omega_{0}\right)>\nu_{k}(\omega)=$ $\nu_{k}\left(\omega_{0}\right)$. Notice that $\nu_{k}(B)=\nu_{k}\left(B-B_{0}\right) \leq \Theta_{k}\left(\delta_{1}\right)$. 
If $\nu_{k}(B)<\Theta_{k}\left(\delta_{1}\right)$ we repeat the procedure again. We claim that after a finite number of steps we obtain $\tilde{\omega}=\tilde{A} d x-\tilde{B} d y \in \mathcal{E}\left(f_{k}\right) \backslash f_{k} \cdot \Omega\left(\log C_{k}\right)$ with $\nu_{k}(\tilde{\omega})=\tilde{\delta}$ and $\Theta_{k}(\tilde{\delta})=\nu_{k}(\tilde{B})=\nu_{k}(B)$.

In fact, if it is not the case we obtain

$$
\omega-\sum_{i=0}^{\infty} \omega_{i}=\left(A-\sum_{i=0}^{\infty} A_{i}\right) d x-\left(B-\sum_{i=0}^{j} B_{i}\right) d y
$$

with $\nu_{k}\left(\omega-\sum_{i=0}^{\infty} \omega_{i}\right)=\infty$ and $\nu_{k}(B)=\nu_{k}\left(B-\sum_{i=0}^{\infty} B_{i}\right)$, that is, $\omega-\sum_{i=0}^{\infty} \omega_{i} \in \mathcal{E}\left(f_{k}\right) \cap$ $f_{k} \cdot \Omega\left(\log C_{k}\right)$. By Proposition 4.1 we must have $\mu_{k}-1+\frac{v_{0}}{e_{k}}-\nu_{k}(B)=\alpha \notin \Lambda_{k}$ that it is a contradiction.

In this way there exists $\tilde{\omega}=\tilde{A} d x-\tilde{B} d y \in \mathcal{E}\left(f_{k}\right) \backslash f_{k} \cdot \Omega\left(\log C_{k}\right)$ with $\nu_{k}(\tilde{\omega})=\tilde{\delta} \geq \nu_{k}(\omega)$ and $\Theta_{k}(\tilde{\delta})=\nu_{k}(\tilde{B})=\nu_{k}(B)$. In particular, $\left.e_{k}(\tilde{(} \delta)-\Theta_{k}(\tilde{\delta})\right) \geq e_{k}\left(\nu_{k}(\omega)-\nu_{k}(B)\right)>\beta_{k+1}$ and, by Remark 4.6, $\nu_{k}(\tilde{\omega}) \in \Lambda_{k} \backslash \Gamma_{k}$. So, $\lambda=v_{k+1}-e_{k} \alpha=\rho_{k}\left(L_{k}^{2}\right) \subset \rho_{k}\left(\Lambda_{k} \backslash \Gamma_{k}\right)$ and we conclude the proof.

As a consequence any branch with $\Gamma=\left\langle v_{0}, \ldots, v_{g}\right\rangle$ and $n_{g}=2$ has the same Tjurina number and it can be expressed by means $\Gamma$.

Theorem 5.2. For any branch $C_{f}$ in the topological class determined by $\Gamma_{f}=\left\langle v_{0}, \ldots, v_{g}\right\rangle$ with $n_{g}=2$ we have that

$\Lambda_{f} \backslash \Gamma_{f}=\rho_{g-1}\left(\Lambda_{g-1} \backslash \Gamma_{g-1}\right) \dot{\cup}\left\{v_{g}-2 \delta, \delta \in \mathbb{N}^{*} \backslash \Lambda_{g-1}\right\} \dot{\cup}\left\{v_{g}+2 \delta ; \delta \in \mathbb{N} \backslash \Gamma_{g-1}\right\}$ and $\sharp\left(\Lambda_{f} \backslash \Gamma_{f}\right)=\mu_{g-1}$.

In particular, we have that $\tau_{f}=\mu_{f}-\mu_{g-1}$.

Proof: Given $\Gamma_{f}=\left\langle v_{0}, \ldots, v_{g}\right\rangle$ with $n_{g}=e_{g-1}=2$ any $v_{g}<\lambda \notin \Gamma_{f}$ can be uniquely expressed as $\lambda=v_{g}+\sum_{i=1}^{g-1} s_{i} v_{i}-s_{0} v_{0}=v_{g}+2\left(\sum_{i=1}^{g-1} s_{i} \frac{v_{i}}{2}-s_{0} \frac{v_{0}}{2}\right)$ with $0 \leq s_{i}<n_{i}$ for $i>0$ and $s_{0} \geq 0$ (see Remark 2.1). In particular, $\sum_{i=1}^{g-1} s_{i} \frac{v_{i}}{2}-s_{0} \frac{v_{0}}{2} \in \mathbb{N} \backslash \Gamma_{g-1}$ and any $v_{g}<\lambda \notin \Gamma_{f}$ is such that $\lambda \in\left\{v_{g}+2 \delta ; \delta \in \mathbb{N} \backslash \Gamma_{g-1}\right\}$, that is, $\left\{\lambda \in \Lambda_{f} \backslash \Gamma_{f} ; \lambda>v_{g}\right\} \subset\left\{v_{g}+2 \gamma ; \gamma \in \mathbb{N} \backslash \Gamma_{g-1}\right\}$. Consequently, by (16) we have $\left\{\lambda \in \Lambda_{f} \backslash \Gamma_{f} ; \lambda>v_{g}\right\}=\left\{v_{g}+2 \gamma ; \gamma \in \mathbb{N} \backslash \Gamma_{g-1}\right\}$.

Now, by the previous proposition we get

$$
\Lambda_{f} \backslash \Gamma_{f}=\rho_{g-1}\left(\Lambda_{g-1} \backslash \Gamma_{g-1}\right) \dot{\cup}\left\{v_{g}-2 \delta, \delta \in \mathbb{N}^{*} \backslash \Lambda_{g-1}\right\} \dot{\cup}\left\{v_{g}+2 \gamma ; \gamma \in \mathbb{N} \backslash \Gamma_{g-1}\right\}
$$

As $\sharp \rho_{g-1}\left(\Lambda_{g-1} \backslash \Gamma_{g-1}\right)=\sharp\left(\Lambda_{g-1} \backslash \Gamma_{g-1}\right)$, and $\sharp\left(\mathbb{N} \backslash \Gamma_{g-1}\right)=\frac{\mu_{g-1}}{2}$, we obtain

$$
\sharp\left(\Lambda_{f} \backslash \Gamma_{f}\right)=\sharp\left(\Lambda_{g-1} \backslash \Gamma_{g-1}\right)+\sharp\left(\mathbb{N}^{*} \backslash \Lambda_{g-1}\right)+\sharp\left(\mathbb{N} \backslash \Gamma_{g-1}\right)=2 \sharp\left(\mathbb{N} \backslash \Gamma_{g-1}\right)=\mu_{g-1} .
$$

In particular, $\tau_{f}=\mu_{f}-\sharp\left(\Lambda_{f} \backslash \Gamma_{f}\right)=\mu_{f}-\mu_{g-1}$. 


\section{References}

[Ab] Abhyankar, S. S., Irreducibility Criterion for Germs of Analytic Function of Two Complex Variables. Advances in Mathematics 74, 190-257 (1989).

[Al] Almirón, P., On the quotient of Milnor and Tjurina numbers for two-dimensional isolated hypersurface singularities. Preprint in Arxiv: 1910.12843 (2019).

[AABM] Alberich-Carramiñana, M., Almirón, P., Blanco, G. and Melle-Hernández, The minimal Tjurina number of irreducible germs of plane curve singularities. To appear: Indiana University Mathematics Journal.

[BHH] Barroso, E. R. G.; Hernandes, M. E. and Iglesias, M. F. H., On the characterization of nondicritical generalized curve foliations. arXiv:2011.12452.

[BK] Brieskorn, E. and Knörrer, H., Plane Algebraic Curves, Birkhäuser Verlag (1986).

[BMT] Briançon, J.; Maisonobe, P. and Torrelli, T., Matrice Magique associée à un germe de coube plane et division par l'idéal Jacobien. Annales de l'institut Fourier 57 (3), 919-954. (2007).

[De] Delorme, C., Sur les Modules des Singularités de Courbes Planes. Bull. Soc. Math. France 106, 417-446 (1978).

[DG] Dimca, A. and Greuel, G.-M., On 1-forms on isolated complete intersection curve singularities. Journal of Singularities 18, 114-118. (2018).

[GH] Genzmer, Y. and Hernandes, M. E., On the Saito basis and the Tjurina Number for Plane Branches. Trans. Amer. Math. Soc. 373, 3693-3707. (2020).

[H] Hefez, A., Irreducible plane curve singularities in Real and Complex Singularities, Dekker Series of Lecture Notes in Pure and Applied Mathematics, Volume 232, D. Mond and M. J. Saia, Editors (2003).

[HH1] Hefez, A. and Hernandes, M.E., Classification of algebroid plane curves with semigroup $\langle 6,9,19\rangle$. Communications in Algebra, 31(8), 3847-3861 (2003).

[HH2] Hefez, A. and Hernandes, M.E., Standard bases for local rings of branches and their modules of differentials. J. Symbolic Comput. 42, 178-191 (2007).

[HH3] Hefez, A. and Hernandes, M.E., The analytic classification of plane branches. Bull. London Math. Soc. 43(2), 289-298 (2011).

[LP] Luengo, I. and Pfister, G., Normal forms and moduli spaces of curve singularities with semigroup $\langle 2 p, 2 q, 2 p q+d\rangle$. Compositio Mathematica 76, (1990) 247-264. 
[M] Milnor, J., Singular Point of Complex Hypersurfaces. Annals of Mathematics Studies, 61, (1968).

[Pol] Pol, D., On the values of logarithmic residues along curves. Annales de I'Institut Fourier 68 (2), (2018) 725-766.

[Pop] Popescu-Pampul, P., Approximate Roots. Valuation theory and its applications, American Mathematical Society 33, (2003) 285-321.

[S] Saito, K., Theory of logarithmic differential forms and logarithmic vector fields. J. Fac. Sci. Univ. Tokyo Sect. IA Math., v. 27, n. 2, (1980) 265-291.

[Y] Yavorski, P., Normal forms and bases of local rings of irreducible germs of functions of two variables. Journal of Soviet Mathematics 50 (1), 1350-1364 (1990).

[Wan] Wang, Z., Monotonic invariants under blowups. To appear: International Journal of Mathematics: doi.org/10.1142/S0129167X20500937.

[Wat] Watari, M., On the Tjurina Number of Plane Curve Singularities, http://www.rimath.saitama-u.ac.jp/lab.jp/fsakai/216.pdf

[Z1] Zariski, O., Studies in Equisingularity I, II and III. Amer. J. Math., (87), 507-536, 972-1006 (1965) and (90), (1970) 961-1023.

[Z2] Zariski, O., Characterization of plane algebroid curves whose module of differentials has maximum torsion. Proc. Nat. Acad. of Sciences, v. 56, n. 3, (1966) 781-786.

[Z3] Zariski, O., Le problème des modules pour les branches planes (Hermann, Paris, 1986) (French); B. Lichtin, The moduli problem for plane branches, University Lecture Series 39 (American Mathematical Society, Providence, RI, 2006) (English).

Abreu, M. O. R. Hernandes, M. E.

osnar@outlook.com_mehernandes@uem.br

Universidade Estadual de Maringá

Maringá - Paraná - Brazil 